\title{
Análisis de presencia y caracterización de la competencia científica y tecnológica en la evaluación externa de $6^{\circ}$ de Educación Primaria.
}

Máster en Investigación e Innovación en currículum y Formación 2016

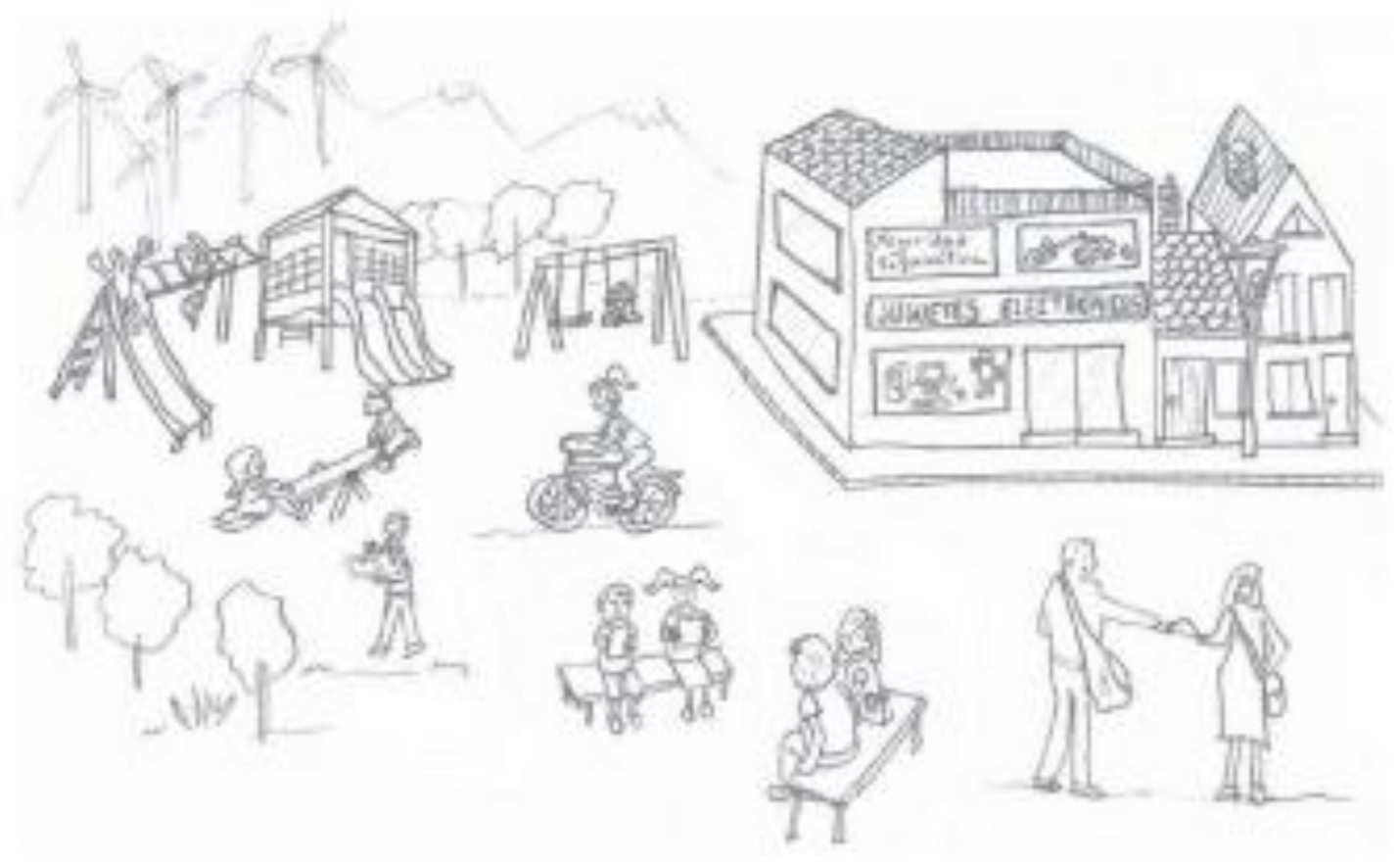





\section{Resumen:}

Esta investigación se centra en el análisis de la "Prueba de la competencia de ciencia y tecnología" elaborada para el curso de $6^{\circ}$ de Primaria por el MECD.

El objetivo es caracterizar las pruebas de evaluación de $6^{\circ}$ de Primaria liberadas, en lo referido a la competencia en Ciencia y Tecnología, de acuerdo a variables extraídas del modelo de la OCDE y del MECD. A través del análisis de la presencia y relaciones de las variables "Contexto", "Demanda cognitiva", "Tipo de respuesta", "Capacidades competenciales” y “Contenido".

Se trata de un estudio cuantitativo exploratorio descriptivo, que utiliza la metodología observacional no participante, los observadores son profesores de Educación Primaria y el instrumento utilizado es una parrilla de observación. La población es la prueba emitida por el MECD.

La distribución de las variables no se ajusta a las demandas emitidas desde sus marcos de referencia, además las relaciones son escasas y siguen sin ajustarse a sus marcos de referencia. Se recomienda ajustar la presencia de las variables y sus relaciones a sus marcos de referencia.

\section{Palabras clave:}

Investigación educativa, evaluación de estudiantes, alfabetización científica, elaboración de test.

\section{Abstract:}

This research focuses on the analysis of the "Science and Technology Literacy test" developed for the $6^{\text {th }}$ of elementary school by MECD.

The aim is to characterize the assessment tests released of $6^{\text {th }}$ Primary, with regard to Science and Technology Literacy, according to variables drawn from OECD and MECD models. Through analysis of the presence and relationships of the variables "Context", "cognitive demand", "Answer type", "Literacy Capabilities" and "Content".

It is a descriptive exploratory quantitative study, using non-participating observational methodology, observers are teachers in elementary education and the instrument used is an observation grid. The population is test issued by the MECD.

The distribution of variables does not comply with the demands issued from their frameworks, further relations are still scarce and still do not fit their frameworks. It is recommended to adjust the presence of the variables and their relationships to their frameworks.

\section{Key words:}

Educational reseach, student evaluation, scientific literacy, competence, test construction. 


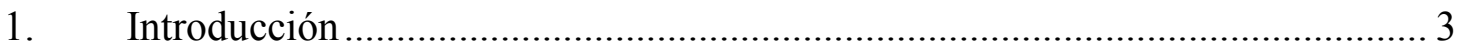

1.1. Aproximación al problema y justificación .................................................... 3

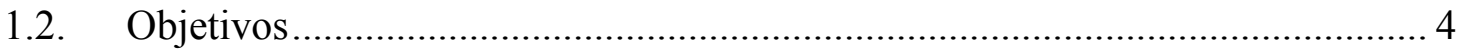

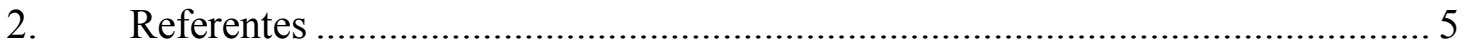

2.1. Marcos referenciales de las pruebas ............................................................ 5

2.2. Competencias Básicas en Ciencia y Tecnología............................................... 7

2.3. El modelo de evaluación externa PISA …................................................. 8

2.4. Modelo de evaluación final de Educación Primaria ........................................... 8

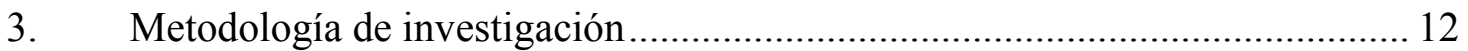

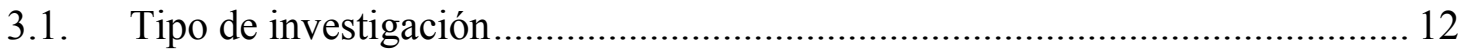

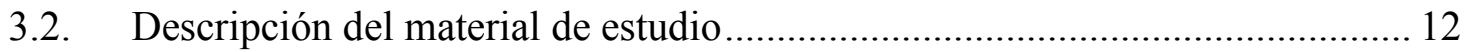

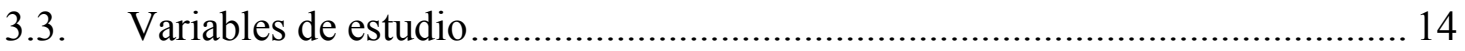

3.4. Instrumento de recogida de datos y procedimiento ........................................ 15

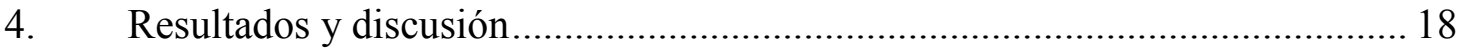

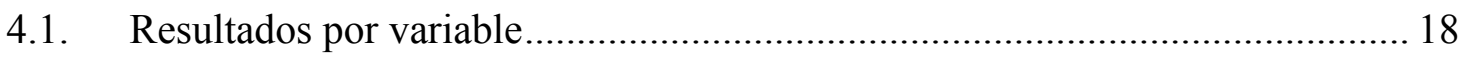

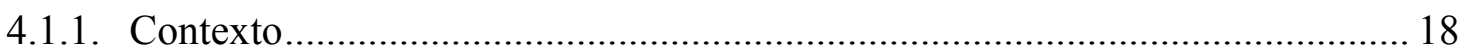

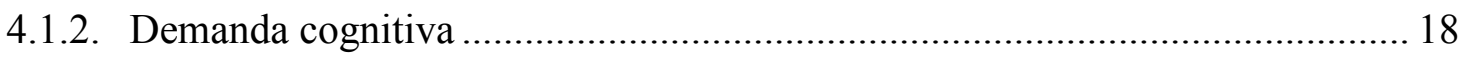

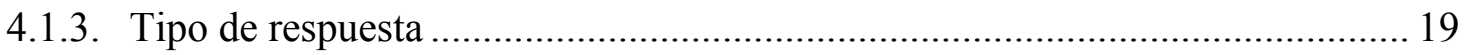

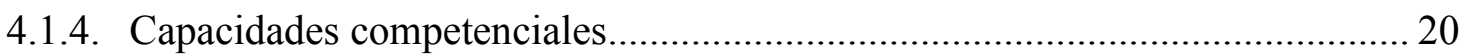

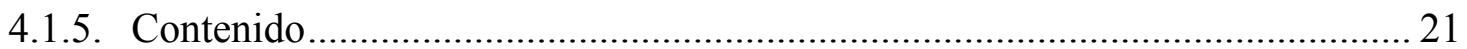

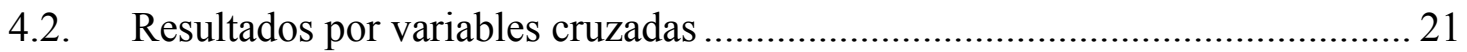

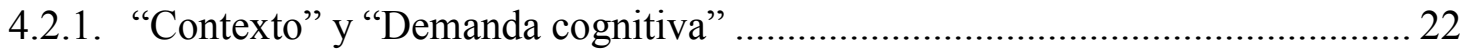

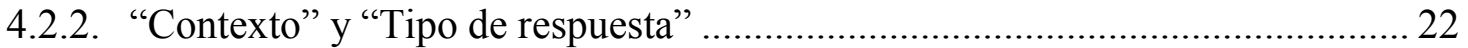

4.2.3. "Contexto" y "Capacidades competenciales" ................................................ 23

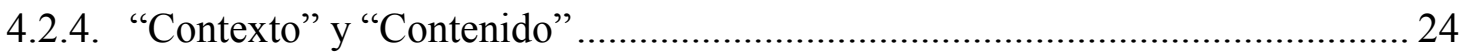

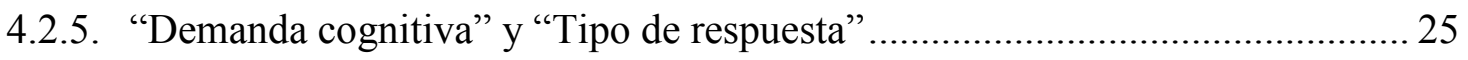

4.2.6. "Demanda cognitiva" y "Capacidades competenciales" ................................. 26 


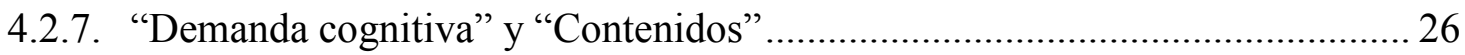

4.2.8. "Tipo de respuesta” y "Capacidades competenciales" .................................... 27

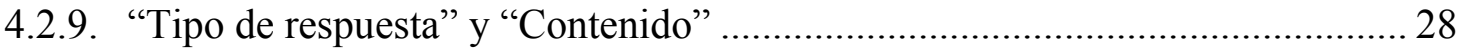

4.2.10. "Capacidades competenciales" y "Contenidos" ........................................... 29

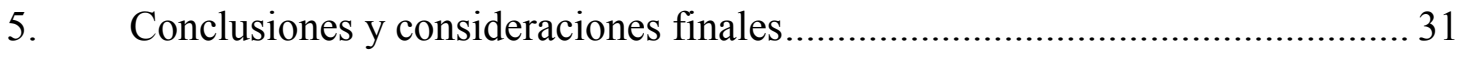

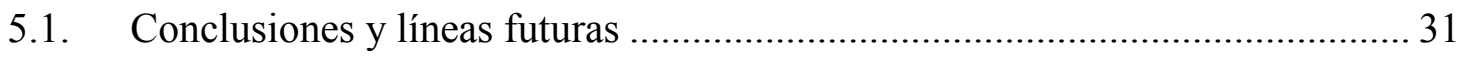

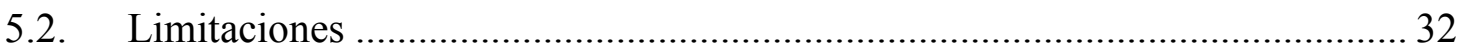

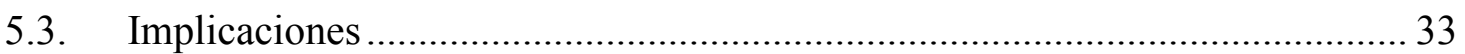

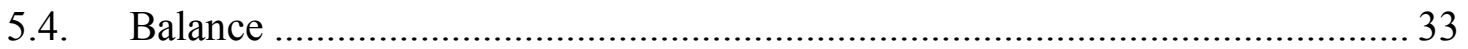

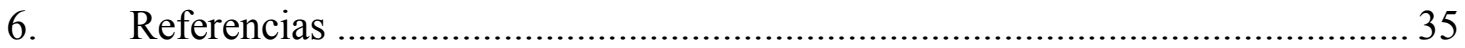




\section{Introducción}

\subsection{Aproximación al problema y justificación}

Este trabajo se centra en las pruebas de evaluación externa de $6^{\circ}$ de Educación Primaria, en particular en el análisis de su idoneidad para la evaluación de la competencia en Ciencia y Tecnología establecida tanto por el Programme for International Student Assessment (PISA) de la Organization for Economic Co-operation and Development (OECD) como por el Ministerio de Educación Cultura y Deporte (MECD).

Para abordar este problema se definen una serie de variables de análisis, que provienen del fundamento teórico de las propias pruebas externas y se centran en el propio contenido, la variedad de contextos en los que se enmarcan los ítems, las demandas cognitivas necesarias para su realización, el tipo de respuesta demandada y las capacidades competenciales que hay que emplear para su resolución.

Son varios los motivos por los que se plantea llevar a cabo esta investigación. El primero es que las pruebas de evaluación externa forman parte de las directrices curriculares de evaluación. La Ley Orgánica 8/2013, de 9 de diciembre, para la mejora de la calidad educativa (LOMCE) establece que la administración educativa debe implantarr evaluaciones externas en los centros educativos.

El segundo motivo es desarrollar una metodología que permita determinar la idoneidad de una evaluación externa para evaluar una competencia, partiendo del hecho de que se trata de pruebas diseñadas para su realización con papel y lápiz, y ordenador en algunos casos. Debido a ello, en nuestro análisis se omitirán los posibles defectos asociados al formato de las mismas, ya admitidos en el Marco General de Evaluación Final de Educación Primaria (MECD, 2015a).

El tercer motivo es que hay investigaciones como Mayorga, Gallardo y Jimeno (2014), Gallardo, Mayorga y Sierra (2014), Yus, Fernández, Gallardo, Barquín, Sepúlveda y Serván (2013) y Gallardo, Fernández, Sepúlveda, Servan, Yus y Barquín (2010) en relación a otras pruebas diagnóstico, signo de la relevancia social y educativa de esta temática. Sin embargo, no hay investigaciones que se centren en las pruebas de evaluación de $6^{\circ}$ de Educación Primaria, lo que justifica el interés y la originalidad de este Trabajo Final de Máster. 
El último motivo, de carácter personal, hace referencia a mi futuro profesional como docente e investigador. Parto de la convicción de que es necesario conocer los procesos de evaluación externos. Si sabemos cómo van a evaluar y qué van a evaluar, podremos utilizar estos conocimientos como uno de los elementos que nos permiten configurar el proceso de enseñanza-aprendizaje de forma coherente, de manera que se pueda desarrollar una evaluación que se ajuste más a sus funciones tal y como las define Maestro (2005): rendir cuentas, informar, comparar y mejorar. Por otro lado, es interesante remarcar que las evaluaciones externas tienen cada vez mayor importancia en nuestros sistemas educativos y ya forman parte indisolubles de los mismos.

\subsection{Objetivos}

El objetivo principal de este TFM es caracterizar las pruebas de evaluación de $6^{\circ}$ de Primaria liberadas, en lo referido a la competencia en Ciencia y Tecnología, de acuerdo a variables extraídas del modelo PISA y del MECD.

Para ello se plantean los dos siguientes objetivos específicos:

1. Evaluar la presencia de las variables en lo referido a la competencia Ciencia y Tecnología, de acuerdo al modelo PISA y al del MECD en las pruebas de evaluación de $6^{\circ}$ de Primaria liberadas.

2. Identificar las relaciones existentes entre las variables de estudio, para si procede, formular propuesta de mejoras. 


\section{Referentes}

En este apartado se presentan los dos referentes que sustentan la organización de este trabajo: el modelo de evaluación final de Educación Primaria propuesto por el Ministerio de Educación, Cultura y Deporte y el modelo propuesto por la OCDE.

\subsection{Marcos referenciales de las pruebas}

La Ley Orgánica 2/2006, de 3 de mayo, de Educación (LOE), hace referencia en su preámbulo a la necesidad de cohesión social, al aprendizaje permanente a lo largo de la vida y a la sociedad del conocimiento, introduciendo el término competencias básicas en la normativa educativa (Ley Orgánica de Educación, 2006). La Ley Orgánica 8/2013, de 9 de diciembre, para la Mejora de Calidad Educativa (LOMCE), continúa con el desarrollo del modelo de currículo basado en competencias e implanta una nueva denominación: competencias clave (Ley Orgánica 8/2013, 2013). Se las define como "aquellas que todas las personas precisan para su realización y desarrollo personal, así como para la ciudadanía activa, la inclusión social y el empleo" (Real Decreto 126/2014, 2014, p. 19350), identificándolas así como esenciales para el bienestar de las sociedades europeas, el crecimiento económico y la innovación.

Más adelante, en su artículo "2. Definiciones”, apartado c) específica que por competencias clave se entienden las "capacidades para aplicar de forma integrada los contenidos propios de cada enseñanza y etapa educativa, con el fin de lograr la realización adecuada de actividades y la resolución eficaz de problemas complejos" (Real Decreto 126/2014, 2014, p. 19351).

Desde la Unión Europea, y más concretamente desde el Consejo Europeo de Lisboa en el año 2000, pasando por las Conclusiones del Consejo de 2009 sobre el Marco Estratégico para la cooperación europea en el ámbito de la educación y la formación ("ET 2020") y hasta nuestros días, se insiste en la necesidad de que los ciudadanos adquieran las competencias clave como condición imprescindible para lograr que alcancen un pleno desarrollo personal, social y profesional que se ajuste a las demandas de un mundo globalizado y haga posible el desarrollo económico, vinculado al conocimiento (Secretaría de Estado de Educación y Formación Profesional, 2011). El Parlamento Europeo y el Consejo establecen la justificación de esa apuesta europea que 
constituirá un marco de referencia para todos los estados miembros en los siguientes términos:

\begin{abstract}
"Dados los nuevos retos que la globalización sigue planteando a la Unión Europea, cada ciudadano requerirá una amplia gama de competencias para adaptarse de modo flexible a un mundo que está cambiando con rapidez y que muestra múltiples interconexiones. En su doble función -social y económica-, la educación y la formación deben desempeñar un papel fundamental para garantizar que los ciudadanos europeos adquieran las competencias clave necesarias para poder adaptarse de manera flexible a dichos cambios" (MECD, 2015b), p. 7).
\end{abstract}

PISA es una adaptación del Proyecto Definition and Selection of Competencies DeSeCo. Los interrogantes esenciales del proyecto son descritos por Rychen y Salganik (2006) en los siguientes términos:
¿QQué competencias y habilidades son relevantes para que un individuo pueda llevar una vida exitosa y responsable y la sociedad logre enfrentar los retos del presente y del futuro? ¿Cuáles son las bases normativas teóricas y conceptuales para definir y elegir un conjunto limitado de competencias clave?" (Rychen y Salgamik, 2006, p. 63)

Un importante referente en este ámbito de las competencias es el que tiene que ver con las evaluaciones internacionales. Así la International Association for the Evaluation of Educational Achievement desarrolla estudios internacionales de evaluación educativa a gran escala desde los años 60 del siglo pasado. PIRLS (Estudio Internacional de Progreso en Comprensión Lectora), TIMSS (Estudio Internacional de Tendencias en Matemáticas y Ciencias) y TIMSS Numeracy evalúan el rendimiento del alumnado de Educación Primaria en diversos países, entre ellos España. PIRLS se encarga evaluar el rendimiento en comprensión lectora, valorando los propósitos y los procesos de comprensión implicados en la misma, así como los hábitos y actitudes ante la lectura. TIMSS y TIMSS Numeracy evalúan el rendimiento en matemáticas, ciencias y tecnología valorando tanto los contenidos de estas áreas como las capacidades cognitivas del alumnado. 
Por último, la OCDE, en su programa PISA enfocado a la Educación Secundaria, plantea que el éxito en la vida de un estudiante dependerá de la adquisición de un rango amplio de competencias. Según PISA (2013a), la competencia, más que un producto adquirido, es un proceso que se va desarrollando a lo largo de toda la vida. Lo importante es que se intenta evaluar no solo si el alumnado puede reproducir un conocimiento, sino también si puede extrapolar lo que han aprendido a situaciones distintas y nuevas. Esta evaluación hace hincapié en la comprensión de los conceptos y en si somos capaces de aplicarlos en diversidad de contextos y situaciones.

\subsection{Competencias Básicas en Ciencia y Tecnología}

Según la Orden ECD/65/2015 y el Real Decreto 129/2014, que describen las relaciones entre las competencias, los contenidos y los criterios de evaluación de la educación primaria, la educación secundaria obligatoria y el bachillerato, las competencias en ciencia y tecnología proporcionan un acercamiento al mundo físico y a la interacción responsable con él desde acciones individuales y colectivas orientadas a la conservación y mejora del medio natural, decisivas para la protección y mantenimiento de la calidad de vida y el progreso de los pueblos. Estas competencias contribuyen al desarrollo del pensamiento científico, incluyendo la aplicación de los métodos propios del área y de las destrezas tecnológicas que conducen a la adquisición de conocimientos, el contraste de ideas y la aplicación de los descubrimientos al bienestar social. Por otra parte, capacitan el desarrollo de juicios críticos sobre los hechos científicos y tecnológicos que se suceden a lo largo de los tiempos, pasados y actuales.

Se requiere igualmente el fomento de destrezas que permitan utilizar y manipular herramientas y máquinas tecnológicas, así como utilizar datos y procesos científicos para alcanzar un objetivo; es decir, identificar preguntas, resolver problemas, llegar a una conclusión o tomar decisiones basadas en pruebas y argumentos.

Asimismo, estas competencias incluyen actitudes y valores relacionados con la asunción de criterios éticos asociados a la ciencia y a la tecnología, el interés por la ciencia, el apoyo a la investigación científica y la valoración del conocimiento científico; así como el sentido de la responsabilidad en relación a la conservación de los recursos naturales y a las cuestiones medioambientales y a la adopción de una actitud adecuada para lograr una vida física y mental saludable en un entorno natural y social. 


\subsection{El modelo de evaluación externa PISA}

El Programme for International Student Assessment (PISA) es considerado como el programa de evaluación más completo sobre el desarrollo de competencias en nuestro alumnado, y como instrumento de recogida de información respecto a sus familias y las instituciones educativas que lo forman (OCDE, 2007). La OCDE define la competencia científica y tecnológica, o Scientific Literacy, en el marco PISA 2015 como el desglose de tres competencias expresadas como capacidades:

"la primera es la capacidad de proporcionar argumentos de fenómenos naturales, artefactos y técnicas tecnológicas y de sus implicaciones para la sociedad. Dicha capacidad requiere un conocimiento de las ideas básicas de la ciencia y los interrogantes que enmarcan la práctica y objetivos de la ciencia. El segundo es la capacidad de utilizar el conocimiento y la comprensión científica para identificar las preguntas que pueden ser respondidas por la investigación científica; identificar si los procedimientos usados son los apropiados y proponer formas para abordar estas cuestiones. La tercera es la competencia para interpretar y evaluar los datos y pruebas científicamente y evaluar si las conclusiones son correctas”. (OCDE, 2013b, p. 5).

\subsection{Modelo de evaluación final de Educación Primaria}

La LOMCE establece en su artículo 6 bis, apartado segundo, letra a), $3^{\circ}$, que el gobierno de España deberá:

"Determinar los criterios de evaluación del logro de los objetivos de las enseñanzas y etapas educativas y del grado de adquisición de las competencias correspondientes, así como las características generales de las pruebas, en relación con la evaluación final de Educación Primaria”. (Ley Orgánica 8/2013, 2013 , p. 97868)

En el artículo 144.2 de la LOMCE se establece que "las Administraciones educativas podrán establecer otras evaluaciones con fines de diagnóstico" y en su artículo 144.3 que "las autoridades establecerán las medidas más adecuadas para que las condiciones de realización de las evaluaciones individualizadas se adapten a las necesidades del 
alumnado con necesidades educativas especiales" (Ley Orgánica 8/2013, 2013, p. 97908).

La LOMCE es la base normativa para el desarrollo de la evaluación final de Educación Primaria, estableciendo su artículo 21 el desarrollo de la misma, según el cual en $6^{\circ}$ de Educación Primaria se procederá a la evaluación de las Competencias Básicas en Ciencia y Tecnología; los criterios de evaluación serán establecidos por el MECD, los resultados de la evaluación se expresarán en niveles, se elaborará un informe y se podrán establecer planes específicos de mejora para los centros con peores resultados.

El Marco General de Evaluación Final de Educación Primaria Ministerio de Educación Ciencia y Deporte, esta evaluación: "completa y enriquece la información y la orientación que reciben los alumnos y alumnas, sus familias y los centros", "proporciona información a los centros educativos, con la finalidad de la revisión de los procesos de enseñanza y de aprendizaje y la mejora educativa a partir de los resultados" y "permite mejorar a través de la disposición de más información y de la retroalimentación entre evaluación y práctica docente, con el objetivo de tomar las decisiones más acertadas, adaptadas a las necesidades y características del contexto de cada centro" (MECD, 2015a, p.. 5).

La evaluación individualizada que, según la LOMCE, han de realizar todo el alumnado al finalizar el sexto curso de Educación Primaria, busca comprobar el grado de adquisición de la competencia en comunicación lingüística, de la competencia Matemática y de las Competencias Básicas en Ciencia y Tecnología, así como el logro de los objetivos de la etapa. Durante este estudio nos centraremos en las Competencias Básicas en Ciencia y Tecnología.

La evaluación requiere del empleo de instrumentos que incluyan unidades de evaluación adecuadas a las Competencias Básicas en Ciencia y Tecnología, que tengan en cuenta situaciones y contextos, concretos y diversos, que permitan al alumnado demostrar su dominio y aplicación de las Competencias Básicas en Ciencia y Tecnología. Todo ello con la premisa de que su implantación debe ser viable.

El aprendizaje del alumnado puede ser evaluado en el aula a través de diferentes técnicas y utilizando instrumentos de evaluación muy diversos. Para evaluar competencias, los métodos que se muestran más adecuados, por viabilidad y 
efectividad, son los que se basan en la valoración de la información obtenida de las respuestas que ofrece el alumnado cuando se enfrenta a situaciones que requieren la aplicación de conocimientos.

El Marco General de la evaluación final de Educación Primaria (MECD, 2015a), pone de manifiesto que este tipo de pruebas presenta un hándicap a la hora de evaluar determinadas competencias, ya que para poder evaluarlas es necesaria la observación directa del desempeño del alumnado, lo que podría hacerse en la evaluación de ciertas habilidades manipulativas, actitudes o valores; es decir, pone en duda la validez de este tipo de pruebas. En general, el grado en que el alumnado ha desarrollado las competencias, según este marco, puede ser determinado mediante procedimientos como la resolución de problemas, la realización de tareas complejas y actividades prácticas, las simulaciones de situaciones reales, la elaboración de portafolios, o mediante la realización de balances de competencias ${ }^{1}$ a partir de la autoevaluación o de entrevistas con el sujeto.

Todas estas estrategias de evaluación resultan complejas o incluso inviables cuando se trabaja con una población de alumnos y alumnas amplia y se pretende garantizar la comparabilidad entre sujetos. Las dificultades que surgen para la implantación de estas técnicas llevan a la utilización de pruebas de evaluación externa administradas colectivamente, que constituyen el procedimiento habitual de las evaluaciones autonómicas, nacionales e internacionales que vienen realizándose sobre el rendimiento del alumnado. Sirven como ejemplo las Pruebas ESCALA a nivel autonómico andaluz, las Pruebas de $3^{\circ}$ y $6^{\circ}$ de Educación Primaria a nivel estatal o las pruebas TIMSS a nivel internacional.

Para llevar a cabo esta evaluación se ha decidido emplear pruebas en las que se combinen diferentes formatos de ítems, basados en situaciones problemáticas, que constituyen las unidades de evaluación. Se trata de pruebas elaboradas a partir de situaciones problemáticas que sirven como interrogantes, y que se acercan a situaciones similares a las que el alumnado puede encontrar en su vida.

En el caso de la prueba que evalúa la competencia científica, las preguntas formuladas a partir de las situaciones problemáticas van dirigidas a comprobar el grado de dominio

\footnotetext{
${ }^{11}$ El balance de competencias es una estrategia para el análisis de los conocimientos, capacidades y competencias de una persona, que incluye su aptitud y su motivación hacia su formación.
} 
de las Competencias Básicas en Ciencia y Tecnología, objeto de la evaluación, y sus componentes observables, como las capacidades necesarias relacionadas con dicha competencia. En cada ítem se presenta una situación problemática que sirve como estímulo para plantear un conjunto de cuestiones que, según el MECD (2015a), se podrían encuadrar en alguno de los siguientes formatos: preguntas de respuesta cerrada; preguntas de respuesta semiconstruida; preguntas de respuesta construida y preguntas de respuesta abierta. No se describen en este apartado al estar incluidas como una de las variables de estudio y, por tanto, serán comentadas posteriormente. 


\section{Metodología de investigación}

Para concretar el tipo de investigación, se define el tipo de investigación, el material de estudio, las variables, el instrumento y el procedimiento.

\subsection{Tipo de investigación}

Esta investigación sigue una metodología cuantitativa que corresponde a un diseño ex post facto, ya que la observación se realiza una vez acabado el suceso. Por otro lado, se trata de una investigación exploratorio-descriptiva. Exploratoria ya que, aunque se ha tomado como referencia la investigación de Mayorga et al. (2014), la población de estudio es diferente y no existen, o por lo menos no se han encontrado, estudios enfocados a esta misma población. $\mathrm{Y}$ de tipo descriptivo porque se llevará a cabo una caracterización de los ítems de las pruebas disponibles, de acuerdo a las variables descritas en el marco PISA (OCDE, 2013a), el Marco General de la evaluación final de Educación Primaria y el Real Decreto 126/2014, de 28 de febrero, por el que se establece el currículo básico de la Educación Primaria.

La técnica de recogida de datos utilizada es la observación no participante, ya que se sigue un plan previamente establecido concentrando la atención en determinados aspectos de las pruebas a estudiar, sin interacción entre el material observado y el observador que derive en una modificación de aquel. Por tanto, se trata de un metodología observacional deductiva en las que las variables a observar vienen definidas desde el marco normativo tomado de referencia.

En cuanto a los criterios de muestreo observacional, nos encontramos con que se trata de conductas momentáneas, ya que el interés se centra en la aparición del rasgo. Por último, estamos hablado de un muestreo focal, al realizar muestras en las que se registran las variables de un material que previamente ha sido seleccionado por ser el "foco" de observación.

\subsection{Descripción del material de estudio}

Esta investigación analizará la totalidad del material de estudio, entendiendo como tal todas las evaluaciones externas susceptibles de análisis. Es decir, las pruebas de 
evaluación de $6^{\circ}$ de Primaria del Ministerio de Educación Ciencia y Deporte que han sido liberadas (MECD, 2015b). ${ }^{2}$

La figura 1 muestra una de las situaciones problemáticas de la prueba.

\section{CUMPLEAÑOS CIENTIFICO}

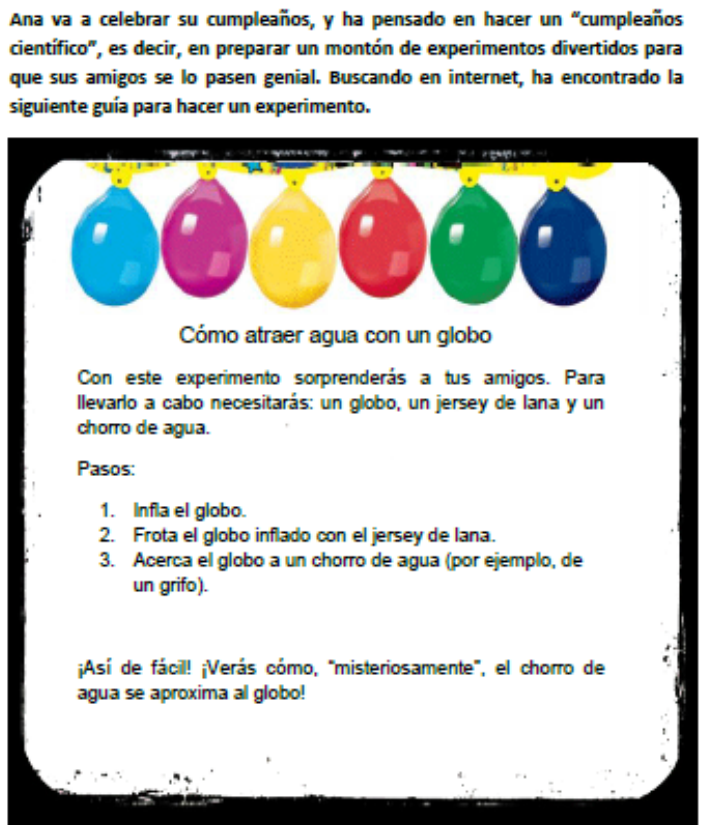

Figura 1. Cumpleaños científico.

La Figura 2 muestra uno de los ítems asociados a la situación presentada en la Figura 1. En él se puede ver cómo se relacionan las preguntas con las situaciones problemáticas iniciales, solicitando al alumnado clasificar fuentes de energía.

\section{CCT16}

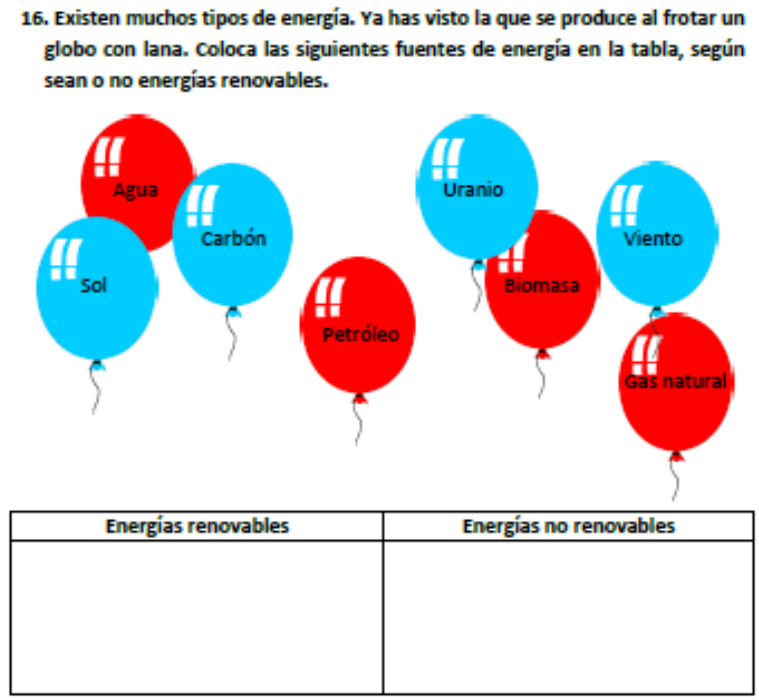

Figura 2. Ítem CCT16.

2 http://www.mecd.gob.es/dctm/inee/evaluacionsextoprimaria/pruebamodelo6epcyt.pdf?documentId= $0901 \mathrm{e} 72 \mathrm{~b} 81 \mathrm{~d} 3 \mathrm{c} 84 \mathrm{f}$ 
La prueba consta de seis situaciones problemáticas que dan lugar a 37 cuestiones relacionadas, que constituyen la muestra de nuestro estudio $(\mathrm{N}=37)$.

\subsection{Variables de estudio}

Las variables analizadas en este estudio son (Tabla 1):

- Los contextos en los que se enmarcan las tareas.

- Los requerimientos de demanda cognitiva necesarios para la realización de la tarea.

- Los tipos de respuestas demandadas desde los interrogantes planteados en la tarea

- Las capacidades competenciales empleadas para su resolución.

- Los bloques de contenido utilizados.

Los contextos aplicables a la prueba analizada están caracterizados y definidos en el Marco General de la evaluación final de Educación Primaria (MECD, 2015a), pudiendo tomar los siguientes valores: "Personal”, "Escolar", "Social” y "Artístico-humanístico.

El requerimiento de demanda cognitiva necesaria para la realización de la tarea esta caracterizado por el Marco General de la evaluación final de Educación Primaria (MECD, 2015a). La variable "Demanda cognitiva" quedaría definida en tres valores que describen los procesos mentales aplicados por el alumnado: "Conocer y reproducir", "Aplicar y analizar" y "Razonar y reflexionar".

La variable "Tipo de respuesta" viene definida desde el Marco General de la evaluación final de Educación Primaria (MECD, 2015a). Los valores asociados a esta variable son: "Cerrada", "Semiconstruida", "Construida" y "Abierta"

La OCDE (2013b) define tres competencias implicadas en el desarrollo de la Competencia científica. Estas tres competencias son incluidas en esta investigación dentro de la variable denominada "Capacidades competenciales". Los valores de esta variable son: "explicar fenómenos científicamente", "evaluar y diseñar investigaciones científicas" e "interpretar investigaciones científicas".

La variable "Contenido" queda caracterizada por el Ministerio de Educación Cultura y Deporte en el R.D. 126/2014 de 28 de febrero, por el que se establece el currículo básico de la Educación Primaria. De este estudio ha sido excluido el bloque "Iniciación 
a la actividad científica" porque así lo establece el Marco General de la evaluación final de Educación Primaria (MECD, 2015a), quedando sus valores de la siguiente manera: "los seres vivos", "el ser humano y la salud", "la materia y la energía" y "la tecnología, los objetos y las máquinas”.

Tabla 1

Variables y valores

\begin{tabular}{cc}
\hline Variables & Valores \\
\hline \multirow{2}{*}{ Contexto } & Personal \\
& Escolar \\
& Social \\
& Artístico humanístico \\
\hline \multirow{2}{*}{ Demanda cognitiva } & Conocer y reproducir \\
& Aplicar y analizar \\
& Razonar y reflexionar \\
\hline Tipo de respuesta & Cerrada \\
& Semiconstruida \\
& Construida \\
Abierta \\
\hline Capacidades competenciales & Evaluar y diseñar investigaciones \\
& científicas \\
& Interpretar datos científicamente \\
\hline Contenidos & Los seres vivos \\
& El ser humano y la salud \\
& La materia y la energía \\
& La tecnología, los objetos y las máquinas \\
\hline
\end{tabular}

En el Anexo 1 se presentan las variables de estudio de un modo más pormenorizado, así como las definiciones de cada uno de sus valores.

\subsection{Instrumento de recogida de datos y procedimiento}

El instrumento de recogida de datos utilizado en esta investigación corresponde a una parrilla de observación de doble entrada. En la primera columna aparecen las denominaciones de cada uno de los ítems de las pruebas analizados, en la primera línea aparecen las variables estudiadas y en la segunda fila aparecen los valores asociados a cada una de las variables. De esta manera se consigue facilitar la observación, ya que en dos hojas se consiguen expresar todos los resultados de las observaciones y facilita la 
localización de los elementos observados y su relación con las variables. En el Anexo 2 pueden encontrar la parrilla de observación utilizada en estas observaciones.

El procedimiento utilizado se ha centrado en el análisis de las pruebas de $6^{\circ}$ de Educación Primaria por grupos de expertos y expertas. Se trata de cuatro expertas y un experto, todos pertenecientes al cuerpo de profesorado de Educación Primaria, funcionarios y funcionarias de carrera, con más de 7 años de experiencia en la etapa educativa. Se buscaron juicios de expertos y expertas ajenos a la investigación para dar una mayor fiabilidad a los resultados, alejándonos de lo realizado en investigaciones, como las de Mayorga et al. (2014), en la que la observación fue realizada por los propios investigadores e investigadoras.

Para facilitar a los observadores el estudio de las pruebas en función de las variables anteriormente definidas se ha creado un sistema de palabras clave (Anexo 3).

Cada experto o experta ha valorado de manera independiente los ítems de cada prueba en función de las variables previamente establecidas. En el Anexo 4 pueden observar un ejemplo del análisis de un ítem. Una vez obtenidos estos resultados se produjo una depuración de los mismos a través de un proceso preestablecido. Dicho proceso consistía en una primera reunión en la que se analizaron las diferencias entre los análisis realizados, de forma que se llegó a una observación conjunta. En caso de que no se hubiera llegado a acuerdo, se llevaría a cabo otra observación individual y una nueva reunión para intentar concretar una respuesta conjunta. Por último, en el caso de que no hubiera acuerdo se pasaría a un experto o experta que sería nombrado de entre los profesores y profesoras del Departamento de Didáctica de las Ciencias Experimentales de la Universidad de Granada para que depurara los defectos de observación.

El procedimiento se agotó en la primera reunión, al llegar a un acuerdo interjueces y juezas; algo ya esperado al tratarse de variables predefinidas para las pruebas. Antes de esta reunión sí había diferencias en las observaciones de los jueces: en la variable "Contexto" aparecían diferencias en el 16\% de los ítems; en la variable "Demanda cognitiva", en el 16\% de los ítems; en la variable "Tipo de respuesta" en el $21 \%$; en la variable "Capacidades competenciales" en el $24 \%$ y en la variable "Contenido" en el $2 \%$ de los ítems analizados. Al finalizar la primera reunión, los expertos y expertas habían alcanzado un acuerdo en las observaciones. 
Esta información fue volcada en el paquete estadístico SPSS v. 22 para su tratamiento estadístico. Para lo que se aplicaron frecuencias, tablas de variables cruzadas y el estadístico "Coeficiente de contingencia de Karl Pearson", la selección de este estadístico se debe a las características del estudio y las variables: no paramétrico y variables nominales. 


\section{Resultados y discusión}

Para mostrar los resultados se seguirá el siguiente orden: en primer lugar se mostrarán los resultados por variables; en segundo lugar, se mostrarán los resultados cruzando las variables para identificar si existe relación entre ellas, profundizando en la descripción del material de estudio y en ambos casos se propondrán mejoras.

\subsection{Resultados por variable}

\subsubsection{Contexto}

En la Tabla 2 se muestran las frecuencias de ítems que se basan en cada contexto, y su porcentaje sobre el total de ítems de la pruebas $(\mathrm{N}=37)$.

Tabla 2

Resultados según contexto

\begin{tabular}{lcc}
\hline & Frecuencia & Porcentaje (\%) \\
\hline Personal & 4 & 11 \\
Escolar & 25 & 68 \\
Social & 8 & 22 \\
Artístico humanístico & 0 & 0 \\
\hline
\end{tabular}

Como se puede observar, la mayoría de ítems se basan en el contexto Escolar (68\%). En cambio, el contexto Personal solo está presente en el 11\% de los ítems, y el Social en el $20 \%$. Por último, se puede observar cómo el contexto Artístico-humanístico no se ve reflejado en ninguno de los ítems.

Esto puede deberse a que el contexto Escolar es un entorno cercano para el alumnado y los docentes, de forma que facilita la tarea de elaboración de los ítems. No obstante, se recomienda una distribución más equitativa entre los contextos ya que, de forma explícita, el Marco General de la evaluación final de Educación Primaria (MECD, 2015a) establece estos cuatro contextos para que el alumnado pueda demostrar la adquisición de las competencias básicas en ciencia y tecnología en estas pruebas.

\subsubsection{Demanda cognitiva}

En la Tabla 3, se muestran se muestran las frecuencias de ítems que se basan en cada demanda cognitiva, y su porcentaje sobre el total de ítems de la pruebas $(\mathrm{N}=37)$. 
Tabla 3

Resultados según demanda cognitiva

\begin{tabular}{lcc}
\hline & Frecuencia & Porcentaje (\%) \\
\hline Conocer y reproducir & 14 & 38 \\
Aplicar y analizar & 10 & 27 \\
Razonar y reflexionar & 13 & 35 \\
\hline
\end{tabular}

La demandas cognitivas "conocer y reproducir" y "razonar y reflexionar" son las más frecuentes (38 y 35\%, respectivamente), siendo "aplicar y analizar" ligeramente menos frecuente $(27 \%)$.

Estudios similares a este, como el llevado a cabo por Gallardo et al. (2014) muestran en su investigación una menor presencia de los niveles de demanda cognitiva superiores similares a "razonar y reflexionar" con un $16 \%$, poniendo de manifiesto que la demanda cognitiva requerida por las pruebas analizadas es mayor. Pero si se acude a las orientaciones dadas por el Marco General de la evaluación final de Educación Primaria (MECD, 2015a), en las que se afirma que lo esperado es que las tres demandas un peso equilibrado en los ítems, nos encontramos con que la prueba analizada no cumple las expectativas marcadas, recomendándose una presencia ponderada de los valores de la variable.

\subsubsection{Tipo de respuesta}

En la Tabla 4, se muestran las frecuencias de ítems que se basan en cada tipo de respuesta, y su porcentaje sobre el total de ítems de la pruebas $(\mathrm{N}=37)$.

Tabla 4

Resultados según tipo de respuesta

\begin{tabular}{lcc}
\hline & Frecuencia & Porcentaje (\%) \\
\hline Cerrada & 9 & 24 \\
Semiconstruida & 19 & 51 \\
Construida & 0 & 0 \\
Abierta & 9 & 24 \\
\hline
\end{tabular}

El tipo de respuesta "Semiconstruida" es el más frecuente (52\%) mientras que los tipos de respuesta "Cerrada" y "Abierta" se dan en menor frecuencia (24\%). Por último destacar que el tipo de respuesta "Construida" no se utiliza en ninguno de los ítems. 
La investigación de Gallardo et al. (2014) establece una diferenciación más simple entre, solo entre "Abierta" (38\%) y "Cerrada" (62\%). Teniendo en cuenta que la categoría "Cerrada" está definida de forma similar en ambos estudios. Se puede afirmar que hay una gran diferencia en los tipos de respuesta observados, presentando la frecuencia del valor "Cerrada" una diferencia del 38\% entre estudios. De forma que la evaluación externa estudiada por Gallardo et al. (2014) se ajusta mejor a los requisitos preestablecidos desde Marco General de la evaluación final de Educación Primaria (MECD, 2015a). Dicho marco establece un mínimo del 40\% de preguntas de respuesta cerradas y un mínimo del $20 \%$ de preguntas de respuestas construidas, semiconstruidas y abiertas, por lo que se puede afirmar que la prueba tampoco cumple con los requisitos preestablecidos para esta variable, recomendándose incluir un mayor número de ítems con el tipo de respuesta "Cerrada" o que se modifique este requisito en el marco de referencia.

\subsubsection{Capacidades competenciales}

En la Tabla 5, se muestran las frecuencias de ítems que se basan en cada capacidad competencial, y su porcentaje sobre el total de ítems de la pruebas $(\mathrm{N}=37)$.

Tabla 5

Resultados según capacidades competenciales

\begin{tabular}{lcc}
\hline & Frecuencia & Porcentaje (\%) \\
\hline Explicar fenómenos científicamente & 16 & 43 \\
Evaluar y diseñar investigaciones científicas & 5 & 14 \\
Interpretar datos científicamente & 16 & 43 \\
\hline
\end{tabular}

La capacidad competencial "Explicar fenómenos científicamente" y la capacidad competencial "Interpretar datos científicamente" son las más frecuentes (ambas con un 43\%). La capacidad competencial "Evaluar y diseñar investigaciones científicas" se da en menor frecuencia (14\%).

Yus et al. (2013) ponen de manifiesto la importancia de estas capacidades competenciales como elementos de la Competencias Básicas en Ciencias y tecnología. Por otro lado, la OCDE (2013) establece que estas tres competencias que nosotros definimos en la variable "Capacidades competenciales" deben ser utilizadas por cualquier persona competente en las Competencias Básicas en Ciencia y Tecnología, de 
lo que se puede deducir que sería interesante que se dieran de manera equitativa o en la mayor presencia posible. Sin embargo, se puede observar como la competencia "Evaluar y diseñar investigaciones científicas" tiene poca presencia en la prueba, recomendándose que se fomente su presencia en la misma.

\subsubsection{Contenido}

En la Tabla 6, se muestran las frecuencias de ítems que se basan en cada contenido, y su porcentaje sobre el total de ítems de la pruebas $(\mathrm{N}=37)$.

Tabla 6

Resultados según contenidos

\begin{tabular}{lcc}
\hline & Frecuencia & Porcentaje (\%) \\
\hline Los seres vivos & 7 & 19 \\
El ser humano y la salud & 9 & 24 \\
La materia y la energía & 10 & 27 \\
$\begin{array}{l}\text { La tecnología, los objetos y las } \\
\text { máquinas }\end{array}$ & 11 & 30 \\
\hline
\end{tabular}

El contenido "La tecnología, los objetos y las máquinas" es el que presenta una mayor frecuencia (30\%). Después le sigue el contenido "La materia y la energía" en frecuencia (27\%). A continuación, el contenido "El ser humano y la salud" presenta una frecuencia del 24\%. El contenido "Los seres vivos" presentaría la menor frecuencia (19\%).

El Real Decreto 126/2014 no establece diferencias entre los bloques de contenido en lo relativo a su importancia. Si es destacable que el Marco General de la evaluación final de Educación Primaria (MECD, 2015a) descarta el contenido "Iniciación a la actividad científica", para las pruebas al considerarlo incluido dentro de los otros bloques de contenido. Por este motivo no se puede considerar su ausencia como una incoherencia de las pruebas, aunque la decisión sí puede ser controvertida. Por otro lado, el resto de contenidos no mantienen un total equilibrio en su presencia en las pruebas, por lo que se recomienda un tratamiento más equilibrado en futuras pruebas.

\subsection{Resultados por variables cruzadas}

Para analizar los resultados por variables cruzadas se ha seleccionado el Coeficiente de contingencia de Karl Pearson, ya que las variables son de tipo nominal, además de tratarse de una prueba no paramétrica. Con este estadístico lo que se busca es 
determinar si las variables están relacionadas, de forma que se podrá establecer cuáles de ellas son dependientes entre sí. Con este estudio se busca identificar posibles relaciones entre variables para describir mejor la prueba y hacer propuestas de mejora.

\subsection{1. "Contexto" y "Demanda cognitiva"}

La Tabla 7 recoge la relación entre la variable "Contextos" y la variable "Demanda cognitiva”.

\section{Tabla 7}

Contextos y Demanda Cognitiva tabulación cruzada

\begin{tabular}{lcccc}
\hline & & \multicolumn{3}{c}{ Demanda Cognitiva } \\
\cline { 3 - 5 } & & \multicolumn{3}{c}{ Aplicar } \\
& & $\begin{array}{c}\text { Conocer y } \\
\text { reproducir }\end{array}$ & $\begin{array}{c}\text { y } \\
\text { analizar }\end{array}$ & $\begin{array}{c}\text { Razonar y } \\
\text { reflexionar }\end{array}$ \\
\hline Contextos & Personal & 5 & 3 & 3 \\
& Escolar & 30 & 22 & 16 \\
& Social & 3 & 3 & 16 \\
& Artístico humanístico & 0 & 0 & 0 \\
\hline
\end{tabular}

El Coeficiente de contingencia de Karl Pearson da un valor de 0,405 y una significación de 0,124 , por lo que no se puede establecer relación estadísticamente significativa entre variables y, por tanto, se consideran variables estadísticamente independientes.

Sin embargo, en la Tabla 7 podemos observar como las demandas cognitivas se dan principalmente en el contexto "Escolar" a excepción del contexto social en el que adquiere relevancia la demanda cognitiva "Razonar y reflexionar". Como ya se argumento anteriormente la variable "Contexto" debería darse de forma equilibrada y la variable "Demanda cognitiva" debería de reflejar equilibrio entre sus valores en los ítems. Por lo cual, se recomienda fomentar un uso más equilibrado de los contextos y las demandas en los ítems, a la hora de elaborar nuevas pruebas.

\subsection{2. "Contexto" $\mathrm{y}$ "Tipo de respuesta"}

La Tabla 8 recoge la relación entre la variable "Contexto" y la variable "Tipo de respuesta”.

Tabla 8

Contextos y Tipo de Respuestas tabulación cruzada 


\begin{tabular}{llcccc} 
& & \multicolumn{4}{c}{ Tipo de Respuestas } \\
\cline { 3 - 6 } Contextos & Perrada & Semiconstruida & Construida & Abierta \\
\hline & Escolar & 0 & 5 & 0 & 5 \\
& Social & 0 & 35 & 0 & 8 \\
& Artístico & & 11 & 0 & 11 \\
& humanístico & 0 & 0 & 0 & 0 \\
\hline
\end{tabular}

El Coeficiente de contingencia de Karl Pearson da un valor de 0,445 y una significación de 0,058 . De forma que no podemos establecer relación entre variables y por lo tanto se trata de variables estadísticamente independientes.

No obstante, en la Tabla 8 muestra cómo el tipo de respuesta cerrada se da solamente en el contexto "Escolar", la semiconstruida se da principalmente en el contexto "Escolar" y la abierta aparece de forma más uniforme. Es destacable que la mayoría de los ítems pertenecen al contexto "Escolar" con tipos de respuestas "Cerradas" y "Semiconstruidas". Por último, se debe mencionar que el contexto "Artístico humanístico" y el tipo de respuesta "Construida" no se han visto reflejadas en la observación. Es recomendable, para futuras pruebas, un uso más equilibrado de las variable "Contexto" y fomentar su relación con el tipo de de respuesta demandada, ajustándolas a las demandas realizadas desde el Marco General de la evaluación final de Educación Primaria (MECD, 2015a).

\subsection{3. "Contexto" y "Capacidades competenciales"}

La Tabla 9 recoge la relación entre la variable "Contexto" y la variable "Capacidades competenciales".

Tabla 9

Contextos y Capacidades competenciales tabulación cruzada

\begin{tabular}{llccc}
\hline & & \multicolumn{3}{c}{ Capacidades competenciales } \\
\cline { 3 - 5 } & & $\begin{array}{c}\text { Explicar } \\
\text { fenómenos } \\
\text { científicamente }\end{array}$ & $\begin{array}{c}\text { Evaluar y } \\
\text { diseñar } \\
\text { investigaciones } \\
\text { científicas }\end{array}$ & $\begin{array}{c}\text { Interpretar } \\
\text { datos } \\
\text { científicamente }\end{array}$ \\
\hline Contextos & Personal & 0 & 5 & 5 \\
& Escolar & 38 & 5 & 24 \\
& Social & 5 & 3 & 24 \\
& Artístico & 0 & 0 & 0 \\
\hline
\end{tabular}


El Coeficiente de contingencia de Karl Pearson da un valor de 0,439 y una significación de 0,066 . Por lo que no se puede establecer relación entre variables y por lo tanto se trata de variables estadísticamente independientes.

Aun así, la Tabla 9 muestra como la capacidad competencial "Explicar fenómenos científicamente" y la capacidad competencial "Interpretar datos científicamente" aparecen principalmente en el contexto "Escolar" y como la capacidad "Evaluar y diseñar investigaciones científicas" se da menos veces y de forma más uniforme. Es destacable que la mayoría de los ítems pertenecen al contexto "Escolar". El contexto "Artístico Humanístico" no se relaciona con ninguno al no haber sido observado en los ítems. Debido a que el marco de referencia fomenta una distribución equilibrada de las variables, al no establecer jerarquía entre sus valores, se recomienda establecer una relación entre variables, en la que se fomente una distribución equilibrada entre ambas variables.

\subsection{4. "Contexto" $\mathrm{y}$ "Contenido"}

La Tabla 10 recoge la relación entre la variable "Contexto" y la variable "Contenido".

Tabla 10

Contextos y Contenidos tabulación cruzada

\begin{tabular}{llcccc}
\hline & & \multicolumn{3}{c}{ Contenidos } \\
\cline { 3 - 6 } & & $\begin{array}{c}\text { Los seres } \\
\text { vivos }\end{array}$ & $\begin{array}{c}\text { El ser } \\
\text { humano y } \\
\text { la salud }\end{array}$ & $\begin{array}{c}\text { La materia } \\
\text { y la } \\
\text { energía }\end{array}$ & $\begin{array}{c}\text { La tecnología, los } \\
\text { objetos y las máquinas }\end{array}$ \\
\hline Contextos & Personal & 0 & 11 & 0 & 0 \\
& Escolar & 16 & 8 & 27 & 16 \\
& Social & 3 & 5 & 0 & 14 \\
& $\begin{array}{c}\text { Artístico } \\
\text { humanístico }\end{array}$ & 0 & 0 & 0 & 0 \\
\hline
\end{tabular}

El Coeficiente de contingencia de Karl Pearson da un valor de 0,605 y una significación de 0,002. Como podemos observar las variables contenidos y contextos si están relacionadas entre sí, por lo que se trata de variables dependientes. 
La Tabla 10 muestra cómo el contenido "La materia y la energía" aparece solamente en el contexto "Escolar", el contenido "Los seres vivos" se dan principalmente en el contexto "Escolar" y no aparece en el "Personal", el contenido "El ser humano y la salud" aparece de forma casi equilibrada en los tres contextos y, por último, el contenido "La tecnología, los objetos y las máquinas" se da en el contexto "Escolar" y "Social".

Se recomienda que se establezcan la máxima diversidad y equilibrio en las variables de cara a futuras evaluaciones de las Competencias Básicas en Ciencia y Tecnología.

\subsection{5. "Demanda cognitiva" $\mathrm{y}$ "Tipo de respuesta"}

La Tabla 11 recoge la relación entre la variable "Demanda cognitiva" y la variable "Tipo de respuesta".

Tabla 11

Demanda Cognitiva y Tipo de Respuestas tabulación cruzada

\begin{tabular}{llcccc}
\hline & & \multicolumn{3}{c}{ Tipo de Respuestas } \\
\cline { 3 - 6 } & Cerrada & Semiconstruida & Construida & Abierta \\
\hline $\begin{array}{l}\text { Demanda } \\
\text { Cognitiva }\end{array}$ & $\begin{array}{l}\text { Conocer y } \\
\text { reproducir }\end{array}$ & 14 & 14 & 0 & 11 \\
& $\begin{array}{l}\text { Aplicar y } \\
\text { analizar }\end{array}$ & 5 & 16 & 0 & 5 \\
& $\begin{array}{l}\text { Razonar y } \\
\text { reflexionar }\end{array}$ & 5 & 22 & 0 & 8 \\
\hline
\end{tabular}

El Coeficiente de contingencia de Karl Pearson da un valor de 0,255 y una significación de 0,642 . No pudiendo establecerse relación entre variables y por lo tanto se trata de variables independientes.

No obstante, la Tabla 11 muestra como el tipo de "Respuesta semiconstruida" es la más utilizada en los ítems analizados, dándose en 19 de los ítems. Por otro lado se puede observar como las demandas cognitivas se dan de forma más uniforme en los totales que en cada una de ellas, ya que, las demandas cognitivas "Aplicar y analizar" y "Razonar y reflexionar" se dan principalmente en las "Respuestas semiconstruidas". 


\subsection{6. "Demanda cognitiva" $\mathrm{y}$ "Capacidades competenciales"}

La Tabla 12 recoge la relación entre la variable "Demanda cognitiva" y la variable "Capacidades competenciales".

Tabla 12

Demanda Cognitiva y Capacidades competenciales tabulación cruzada

\begin{tabular}{llccc}
\hline & & \multicolumn{3}{c}{ Capacidades competenciales } \\
\cline { 3 - 5 } & & $\begin{array}{c}\text { Explicar } \\
\text { fenómenos } \\
\text { científicamente }\end{array}$ & $\begin{array}{c}\text { Evaluar y } \\
\text { diseñar } \\
\text { investigaciones } \\
\text { científicas }\end{array}$ & $\begin{array}{c}\text { Interpretar } \\
\text { datos } \\
\text { científicamente }\end{array}$ \\
\hline $\begin{array}{l}\text { Demanda } \\
\text { Cognitiva }\end{array}$ & $\begin{array}{l}\text { Conocer y } \\
\text { reproducir } \\
\text { Aplicar y } \\
\text { analizar } \\
\text { Razonar y } \\
\text { reflexionar }\end{array}$ & 16 & 0 & 22 \\
\hline
\end{tabular}

El Coeficiente de contingencia de Karl Pearson da un valor de 0,452 y una significación de 0,050 , pudiendo afirmarse que existe relación entre ambas variables y por lo tanto son variables dependientes.

Aún así, en la Tabla 12 la demanda cognitiva "Conocer y reproducir" solo se da en las capacidades competenciales "Explicar fenómenos científicamente" e "Interpretar datos científicamente". La demanda cognitiva "Aplicar y analizar" en la capacidad competencial "Explicar fenómenos científicamente". Y la demanda cognitiva "Razonar y reflexionar" en la capacidad competencial Interpretar datos científicamente.

Se trata de dos variables dependientes, y por tanto no se ajusta a sus marcos de referencia, pues estos recomiendan que se debe fomentar una distribución equilibrada de las capacidades competenciales y las demandas cognitivas en los ítems.

\subsection{7. "Demanda cognitiva" $\mathrm{y}$ "Contenidos"}

La Tabla 13 recoge la relación entre la variable "Demanda cognitiva" y la variable "Contenidos".

Tabla 13

Demanda cognitiva y Contenidos tabulación cruzada 


\begin{tabular}{|c|c|c|c|c|c|}
\hline & & \multicolumn{4}{|c|}{ Contenidos } \\
\hline & & $\begin{array}{l}\text { Los seres } \\
\text { vivos }\end{array}$ & $\begin{array}{c}\text { El ser } \\
\text { humano y } \\
\text { la salud }\end{array}$ & $\begin{array}{c}\text { La materia } \\
\text { y la } \\
\text { energía }\end{array}$ & $\begin{array}{c}\text { La } \\
\text { tecnología, } \\
\text { los objetos y } \\
\text { las máquinas }\end{array}$ \\
\hline \multirow[t]{3}{*}{$\begin{array}{l}\text { Demanda } \\
\text { Cognitiva }\end{array}$} & $\begin{array}{l}\text { Conocer y } \\
\text { reproducir }\end{array}$ & 5 & 16 & 8 & 8 \\
\hline & $\begin{array}{l}\text { Aplicar y } \\
\text { analizar }\end{array}$ & 3 & 3 & 11 & 11 \\
\hline & $\begin{array}{l}\text { Razonar y } \\
\text { reflexionar }\end{array}$ & 11 & 5 & 8 & 11 \\
\hline
\end{tabular}

El Coeficiente de contingencia de Karl Pearson da un valor de 0,382 y una significación de 0,387 , de forma que no podemos establecer relación entre variables y por lo tanto se trata de variables independientes.

No obstante, la Tabla 13 muestra que la demanda cognitiva "Conocer y reproducir" aparece mayoritariamente relacionada con el contenido de "El ser humano y la salud"; la demanda cognitiva "Aplicar y analizar", con los contenidos "La materia y la energía" y "La tecnología, los objetos y las máquinas"; y la demanda cognitiva "Razonar y reflexionar", con los contenidos "Los seres vivos" y "La tecnología, los objetos y las máquinas". Se debe distribuir de manera más equilibrada la variable "Contenidos" y fomentar la distribución equilibrada de los valores de la variable "demanda Cognitiva" con cada uno de los ítems, como demanda el marco de referencia para las variables.

\subsection{8. "Tipo de respuesta" $\mathrm{y}$ "Capacidades competenciales"}

La Tabla 14 recoge la relación entre la variable "Tipo de respuesta" y la variable "Capacidades competenciales".

Tabla 14

Tipo de Respuestas y Capacidades competenciales tabulación cruzada

\begin{tabular}{llccc}
\hline & & \multicolumn{3}{c}{ Capacidades competenciales } \\
\cline { 3 - 5 } & & $\begin{array}{c}\text { Explicar } \\
\text { fenómenos } \\
\text { científicamente }\end{array}$ & $\begin{array}{c}\text { Evaluar y } \\
\text { diseñar } \\
\text { investigaciones } \\
\text { científicas }\end{array}$ & $\begin{array}{c}\text { Interpretar } \\
\text { datos } \\
\text { científicamente }\end{array}$ \\
\hline Tipo de & Cerrada & 8 & 0 & 16 \\
Respuestas & Semiconstruida & 27 & 8 & 16 \\
& Construida & 0 & 0 & 0 \\
\hline
\end{tabular}


El Coeficiente de contingencia de Karl Pearson da un valor de 0,324 y una significación de 0,363 , por lo cual no existe relación entre variables y se trata de variables independientes.

Por otro lado, la Tabla 14 muestra que los tipos de respuesta "Semiestructuradas" aparecen asociadas a las capacidades competenciales "Explicar fenómenos científicamente" e "Interpretar datos científicamente" principalmente y las cerradas a "Explicar fenómenos científicamente" e "Interpretar datos científicamente". La respuesta abierta se presenta de forma más proporcionada. Por último, el tipo de respuesta Construida no aparece en la prueba observada. Como se puede observar, la distribución de las variables no se ajusta a las demandas establecidas desde el marco de referencia, por lo que se recomienda relacionar las variables para que la variable "Capacidades competenciales" aparezca de forma más equilibrada y el tipo de respuesta demandada parezca dentro de las capacidades competenciales ajustada a los parámetros establecidos desde Marco General de la evaluación final de Educación Primaria (MECD, 2015a).

\subsection{9. "Tipo de respuesta" $\mathrm{y}$ "Contenido"}

La Tabla 15 recoge la relación entre la variable "Tipo de respuesta" y la variable "Contenido".

Tabla 15

Tipo de Respuestas y Contenidos tabulación cruzada

\begin{tabular}{llcccc}
\hline & \multicolumn{4}{c}{ Contenidos } \\
\cline { 3 - 6 } & & $\begin{array}{c}\text { Los seres } \\
\text { vivos }\end{array}$ & $\begin{array}{c}\text { El ser } \\
\text { humano y } \\
\text { la salud }\end{array}$ & $\begin{array}{c}\text { La materia } \\
\text { y la } \\
\text { energía }\end{array}$ & $\begin{array}{c}\text { La } \\
\text { tecnología, } \\
\text { los objetos y } \\
\text { las máquinas }\end{array}$ \\
\hline Tipo de & Cerrada & 11 & 3 & 11 & 0 \\
Respuestas & Semiconstruida & 8 & 11 & 14 & 19 \\
& Construida & 0 & 0 & 0 & 0 \\
& Abierta & 0 & 11 & 3 & 11 \\
\hline
\end{tabular}


El Coeficiente de contingencia de Karl Pearson da un valor de 0,505 y una significación de 0,049 , de forma que las variables Tipo de respuesta y contenidos si están relacionadas entre sí y por lo tanto son dependientes.

No obstante, la Tabla 15 muestra que los tipos de "Respuesta semiestructuradas" aparecen asociadas a todos los contenidos aunque mayoritariamente al contenido "La tecnología, los objetos y las máquinas". Los tipos de "Respuesta cerrada" se asocian a los contenidos "Los seres vivos" y "La materia y la energía". Los tipos de "Respuesta abierta" se dan con los contenidos "El ser humano y la salud" y "La tecnología, los objetos y las máquinas", mientras que se puede observar cómo los tipos de respuesta "Construida" no se ven reflejados en la Tabla 19 al no haber sido observados. Este es un caso de dependencia entre variables claro, ya que ambas están definidas por el Marco General de la evaluación final de Educación Primaria (MECD, 2015a). Es lógico pensar que ambas fueron definidas con anterioridad a la elaboración de las pruebas. La variable "Contexto" no tiene ningún tipo de jerarquización entre sus valores o niveles y por lo tanto su distribución debería ser equilibrada, mientras que la variable "Tipo de Respuesta" tiene predefinidas las frecuencias de sus valores. En este caso se recomienda que se mantenga dicha dependencia, pero aplicando la distribución de establecida desde Marco General de la evaluación final de Educación Primaria (MECD, 2015a) a la variable "Tipo de respuesta" así como que se fomentara una distribución más equilibrada en los contenidos.

\subsubsection{0. "Capacidades competenciales" y "Contenidos"}

La Tabla 16 recoge la relación entre la variable "Capacidades competenciales" y la variable "Contenidos".

Tabla 16

Capacidades competenciales y Contenidos tabulación cruzada

\begin{tabular}{llcccc}
\hline & \multicolumn{4}{c}{ Contenidos } \\
\cline { 3 - 6 } & $\begin{array}{c}\text { Los seres } \\
\text { vivos }\end{array}$ & $\begin{array}{c}\text { El ser } \\
\text { humano y } \\
\text { la salud }\end{array}$ & $\begin{array}{c}\text { La materia } \\
\text { y la } \\
\text { energía }\end{array}$ & $\begin{array}{c}\text { La } \\
\text { tecnología, } \\
\text { los objetos } \\
\text { y las } \\
\text { máquinas }\end{array}$ \\
\hline $\begin{array}{l}\text { Capacidades } \\
\text { competenciales }\end{array}$ & $\begin{array}{l}\text { Explicar } \\
\text { fenómenos } \\
\text { científicamente }\end{array}$ & 5 & 3 & 16 & 19 \\
\hline
\end{tabular}




$\begin{array}{lllll}\begin{array}{l}\text { Evaluar y } \\ \text { diseñar } \\ \text { investigaciones } \\ \text { científicas }\end{array} & 0 & 5 & 3 & 5 \\ \begin{array}{l}\text { Interpretar } \\ \text { datos } \\ \text { científicamente }\end{array} & 14 & 16 & 8 & 5\end{array}$

El Coeficiente de contingencia de Karl Pearson da un valor de 0,468 y una significación de 0,110 , no pudiéndose establecer relación estadísticamente significativa entre variables y, por tanto, se consideran variables independientes.

Al observar la Tabla 16 podemos definir que las capacidades competenciales asociadas a un mayor número de ítems son "Explicar fenómenos científicamente" e "Interpretar datos científicamente". Dentro de "Explicar fenómenos científicamente" los contenidos más importantes serían "La materia y la energía" y "La tecnología, los objetos y las máquinas". Tomando como referencia la capacidad competencial "Interpretar datos científicamente" los bloques de contenido con mayor importancia serían "Los seres vivos" y "El ser humano y la sociedad". Por último, si tomamos en consideración la capacidad competencial "Evaluar y diseñar investigaciones" vemos que no se ve representada el contenido "Los seres vivos" y se ve de forma similar en el resto de contenidos. Sería recomendable que se estableciera una relación de equilibrio entre los valores de las dos variables, de forma que se ajustara a sus marcos de referencia. 


\section{Conclusiones y consideraciones finales}

\subsection{Conclusiones y líneas futuras}

Esta investigación se plantea para caracterizar las pruebas de evaluación de $6^{\circ}$ de Educación Primaria, en relación a su marco de referencia, en lo relativo a la competencia Científica y Tecnológica. Según los resultados obtenidos y tal como se ha mostrado en la discusión por variables, nos encontramos con una situación de diversidad en su presencia en las pruebas.

En la variable "Contexto" nos encontramos con una distribución desequilibrada de los utilizados. Se puede observar que el contexto "Escolar" tiene una presencia mayoritaria y sin embargo, el contexto "Artístico-humanístico" no tiene representación en las mismas, algo que choca con el Marco de referencia establecido por el MECD (2015a) y pone en duda la adecuación al mismo.

El mismo marco de referencia establece que las demandas cognitivas se deben de tener un peso ponderal en los ítems. La realidad es que su distribución no es equilibrada y no se ajusta a lo preestablecido por el MECD (2015a).

Por otro lado, el MECD (2015a) también establece la distribución de los tipos de respuestas solicitadas, un mínimo del $40 \%$ de preguntas de respuesta cerradas y un mínimo del $20 \%$ de preguntas de respuestas construidas, semiconstruidas y abiertas. Algo que difiere de la realidad observada, las respuestas cerradas suponen solo el $24 \%$ de los ítems analizados, por lo que se puede afirmar que esta variable no se ajusta a su marco de referencia.

Las capacidades competenciales se ven representadas en su totalidad en las pruebas pero no de una forma equilibrada. Ya que estas competencias o capacidades competenciales son imprescindibles para el dominio de las Competencias Básicas en Ciencia y Tecnología, se puede deducir que todas tienen la misma importancia, de forma que el reparto más lógico sería uno equilibrado, pero en este caso no se puede afirmar que no se ajusta a lo establecido desde la OCDE (2013) al no estar ninguna de ellas excluidas de las observaciones.

El marco de referencia de la variable "Contenido" es el Real Decreto 126/2014 y el Marco General de la evaluación final de Educación Primaria (MECD, 2015a). En este 
caso pasa igual que en las capacidades competenciales, ya que aparecen todos sus niveles pero la distribución no es equilibrada, por lo que no se puede afirmar que no se ajuste a su marco de referencia, pero si sugerir una distribución más equilibrada de sus valores o niveles.

Por otro lado, solo cinco de las variables presentan una relación de dependencia con otras. Se ha podido observar relaciones estadísticamente significativas en tres casos:

- La variable "Contexto" y la variable "Contenido".

- La variable "Demanda cognitiva" y la variable "Capacidades Competenciales".

- La variable "Tipo de respuesta" y la variable "Contenido".

La principal, repercusión que pueden tener estas relaciones es la importancia de establecer criterios previos para la asociación de las mismas a los ítems y entre sí. Siendo deseable que estas relaciones de independencia estadística se amplíen al resto de variables.

Como línea futura para esta investigación sería interesante prolongar longitudinalmente el estudio para analizar si la significación cambia al aumentar el número de datos registrados.

\subsection{Limitaciones}

Esta investigación presenta una limitación y es la propia selección de las variables. Esto se debe a que las variables de estudio presentan diferencias según el marco de referencia seleccionado para el estudio de las mismas. Para solucionar esta limitación se ha cogido el marco de referencia definido para las pruebas y se ha completado con marcos de referencia más generales, en el caso de necesitar alguna caracterización. Como es el caso de la variable "Contenido" con el Real Decreto 126/2014 y el Marco General de la evaluación final de Educación Primaria.

Otra limitación es que no se puede llevar a cabo un estudio longitudinal de las pruebas al solo disponer de una prueba de evaluación. Esta limitación se verá resuelta con los sucesivos análisis previstos durante los próximos cinco años. 


\subsection{Implicaciones}

Las pruebas de evaluación externa, pese a las limitaciones que se indican en los propios marcos (MECD, 2013a) y que algunos autores señalan (Gallardo et al. 2014), son una buena herramienta para determinar la competencia del alumnado y evaluar las necesidades de los centros, ya que permiten su aplicación en grandes poblaciones sin suponer un coste excesivo. Para que esto surta efecto, se deben de elaborar de una forma más sistemática. Es aconsejable definir las variables asociadas a la competencia a evaluar, con anterioridad a las pruebas, en sus marcos de referencia, labor que deben realizar los organismos emisores (OCDE, MECD...). Además, se deben aplicar de forma que permita comprender el nivel competencial del alumnado y controlar al máximo variables extrañas.

Las pruebas de evaluación de $6^{\circ}$ de Educación Primaria sí tenían definidas estas variables, pero a la hora de concretar los ítems se deberían haber tenido en cuenta con más precisión dichas definiciones. Como se ha mostrado, las variables no siempre se ajustan a sus marcos de referencia o al menos se puede mejorar su integración en los ítems.

En cualquier caso, esta investigación pone de manifiesto que la evaluación de la competencia científica y tecnológica llevada a cabo en las pruebas de evaluación de $6^{\circ}$ de Educación Primaria no trata de forma adecuada las variables analizadas. Parece responder más a la facilidad para crear los ítems que a la propia evaluación de la competencia. Por ello, se recomienda que se tengan en cuenta, estas variables, de cara a futuras evaluaciones.

También nos sirve como orientación para saber qué se demanda al alumnado y al profesorado en dicha evaluación externa, como recurso para determinar posibles debilidades en el proceso de enseñanza y aprendizaje o para implantar planes de mejora más concretos.

\subsection{Balance}

Este estudio analiza un instrumento de evaluación externo y realiza una investigación educativa. Con esto he podido continuar con un modelo de trabajo, ya comenzado en mi TFG. Me gustaría pensar que esta investigación servirá a terceras personas, 
convirtiéndome así en el emisor de la situación comunicativa. Como se suele decir, compartir es amar.

Considero que se trata de una investigación de relevancia para mi futuro docente e investigador, ya que me permitirá realizar evaluaciones más completas y premeditadas. También permite adaptar evaluaciones a las exigencias que demandan desde la administración educativa. Y, por último, pone de manifiesto una actitud crítica ante las pruebas de evaluación externas y su adecuación, ya que esta investigación pone de manifiesto que la prueba de evaluación externa analizada tiene margen de mejora respecto a las variables estudiadas y sus relaciones. 


\section{Referencias ${ }^{\mathrm{i}}$}

Gallardo Gil, M., Fenández Navas, M., Sepúlveda Ruiz, M.P., Serván, M., Yus, R., \& Barquín, J. (2010). PISA y la Competencia Científica: Un analisis de las pruebas de PISA en el área de ciencia. Relieve, 16(2), 1-17. Retrieved from http://www.uv.es/RELIEVE/v16n2/RELIEVEv16n2_6.htm

Ley Orgánica de Educación (LOE) (Ley Orgánica 2/2006, de 3 de mayo). Boletín Oficial del Estado, no 106, 2006, 4 mayo. Recuperado de: https:/www.boe.es/ buscar /pdf/2006/BOE-A-2006-7899-consolidado.pdf

Ley orgánica para la mejora de la calidad educativa (LOMCE) (Ley Orgánica 8/2013, 9 de diciembre). Boletín Oficial del Estado, $\mathrm{n}^{\circ}$ 295, 2013, 10 diciembre. Recuperado de: https://www.boe.es/diario_boe/txt.php?id=BOE-A-2013-12886

Maestro, C. (2005). Las evaluaciones nacionales e internacionales. La mejora de la calidad del sistema educativo: el éxito de todos los alumnos como objetivo. Trabajo presentado en Seminario de Primavera 2005: La enseñanza de las Matemáticas y el Informe PISA. Madrid: Fundación Santillana.

Gallardo Gil, M., Mayorga Fernández, M.J. y Sierra Nieto, J.E. (2014). La competencia de 'conocimiento e interacción con el mundo físico y natural': Análisis de las pruebas de evaluación de diagnóstico de Andalucía. Revista Eureka sobre Enseñanza y Divulgación de las Ciencias, 11 (2), 160-180. DOI: 10498/15973

Mayorga Fernández, M.J., Gallardo Gil, M., Jimeno Pérez, M. (2014). Evaluación Diagnóstica en Andalucía: Una investigación del área «competencia matemática». Aula Abierta, 43, 47-52. http://doi.org/10.1016/j.aula.2014.10.002

Ministerio de Educación Ciencia y Deporte. (2015a). Marco General de la evaluación final de Educación Primaria. Recuperado de: http://educalab.es/documents/10180/ 477322/marco-teorico-evaluacion-final-6ep.pdf/4f3c3af0-5f88-4727-88a7-24956e $8 \mathrm{ec} 24 \mathrm{f}$

Ministerio de Educación Ciencia y Deporte. (2015b). Prueba de la competencia en ciencia y tecnología. Recuperado de: http://www.mecd.gob.es/dctm/inee/evalua cionsextoprimaria/pruebamodelo6epcyt.pdf?documentId $=0901 \mathrm{e} 72 \mathrm{~b} 81 \mathrm{~d} 3 \mathrm{c} 84 \mathrm{f}$ 
Orden por la que se describen las relaciones entre las competencias, los contenidos y los criterios de evaluación de la educación primaria, la educación secundaria obligatoria y el bachillerato (Orden ECD/65/2015, de 21 de enero). Boletín Oficial del Estado, $\mathrm{n}^{\circ} 25,2015$, de 29 de enero. Recuperado de: https://www.boe.es/diario _boe/txt.php?id=BOE-A-2015-738

Organización para la Cooperación y el Desarrollo Económicos. (2007). El programa PISA de la OCDE Que es y para que sirve. Paris: OCDE. Recuperado de: http://www.oecd.org/pisa/39730818.pdf

Organización para la Cooperación y el Desarrollo Económicos. (2013a). Marcos y pruebas de evaluación de PISA 2012 Matemáticas , Lectura y Ciencias. Paris: OCDE. Recuperado de: http://www.mecd.gob.es/dctm/inee/internacional/pisa2012 /marco pisa2012.pdf?documentId=0901e72b8177328d

Organización para la Cooperación y el Desarrollo Económicos. (2013b). Pisa 2015 Draft Science Framework. Paris: OCDE. Recuperado de: https://www.oecd.org /pisa/pisaprodu cts/Draft PISA 2015 Science Framework .pdf

Real Decreto por el que se establece el currículo básico de la Educación Primaria (Real Decreto 126/2014, de 28 de febrero). Boletín Oficial del Estado, n 52, 2014, de 1 de marzo. Recuperado de: http://www.boe.es/boe/dias/2014/03/01/pdfs/BOE-A2014-2222.pdf

Rychen, D.S. y Salganik L.H. (2006). Las competencias clave para el bienestar personal, social y económico. Archidona (Málaga): Ediciones Aljibe.

Secretaría de Estado de Educación y Formación Profesional. (2011). Objetivos Educativos Europeos y Españoles Estrategia Educación y Formación 2020. Madrid: Ministerio de Educación. Recuperado de: http://www.mecd.gob.es/dctm /ievaluacion/indicadores-educativos/objetivos-et2020-informe-2011.pdf?document Id $=0901$ e72b80faaff5

Watson, J.M. y Callingham, R. (2003). Statistical literacy: A complex hierarchical construct. Statistics Education Research Journal, 2 (2), 3-46.

Yus Ramos, R., Fernández Navas, M., Gallardo Gil, M., Barquín Ruiz, J., Sepúlveda Ruiz, M.P. y Serván Núñez, M.J. (2013). La competencia científica y su 
evaluación . Análisis de las pruebas estandarizadas de PISA. Revista de Educación, 360, pp. 557-576. http://doi.org/10.4438/1988-592X-RE-2011-360-127

\footnotetext{
${ }^{\mathrm{i}}$ Los enlaces de las referencias fueron comprobados el día 14 de junio de 2016.
} 


\section{Anexos}

1. Descripción de las variables de estudio

2. Instrumento y resultados

3. Sistema de variables

4. Ejemplo del análisis en un ítem (tarea) específica 


\section{Descripción de las variables de estudio}

Las variables de tarea que se analizarán durante este estudio serán los contextos en los que se enmarcan las tareas, los requerimientos de demanda cognitiva necesarios para la realización de la tarea y los tipos de respuestas demandadas desde los interrogantes planteados en la tarea.

La variedad de contextos en las tareas planteadas es algo que se pone de manifiesto desde la OCDE y en la legislación educativa de España a la hora de hablar de competencia científica. Como afirman Watson y Callingham (2003), debe ser valorada la capacidad de trabajar en un determinado contexto para asignar exigencias adicionales en la resolución del problema, y debemos tener en cuenta que las tareas son planteadas desde situaciones problemáticas que necesitan ser resueltas, de lo cual podemos entender que no se puede ser competente en ciencias si no se pueden aplicar en una diversidad de contextos. Para este estudio se utilizará la definición dada de los mismos por el MECD (2016):

- "Personal: englobando problemas o desafíos a los que podría enfrentarse el alumno relacionados con él mismo, su familia o su grupo de amigos.

- Escolar: situaciones relacionadas con la vida escolar y el grupo o grupos de compañeros.

- Social: situaciones referidos al barrio, a la localidad o a la sociedad en general.

- Artístico y humanístico: relacionado con la aplicación de la ciencia y la tecnología al mundo artístico y de las ciencias sociales.” (MECD, 2015, pág. 94).

De esta forma ya tendríamos definida la primera variable "Contexto" y sus categorías "Personal”, "Escolar", "Social” y "Artístico humanístico".

En cuanto al requerimiento de demanda cognitiva necesaria para la realización de la tarea, también me basaré en la caracterización realizada por Marco General de la evaluación final de Educación Primaria (MECD, 2015a). Así la clasificación de la demanda cognitiva quedaría definida en tres dominios que describen los procesos mentales aplicados por el alumnado: 
- "Conocer y reproducir, hace referencia a la habilidad del alumno para recordar, reconocer y describir los hechos, conceptos y procedimientos que son necesarios para unos sólidos fundamentos científicos.

- Aplicar y analizar, se centra en el uso de este conocimiento para generar explicaciones y resolver problemas prácticos.

- Razonar y reflexionar, incluye el uso de las evidencias y de la comprensión científica para analizar, sintetizar y generalizar, a menudo en situaciones no familiares y contextos complejos.” (MECD, 2015, pág. 9495).

Dentro de estos valores se establecen niveles de gradación, pero al tratarse de un estudio exploratorio y no dar más información acerca de las demandas cognitivas no serán tenidos en cuenta para su análisis. Además, si tomamos como referencia la OCDE (2015) nos encontramos con que hacen una caracterización similar a esta pero con la denominación baja demanda cognitiva, media demanda cognitiva y alta demanda cognitiva y sin establecer niveles de gradación internos.

Para caracterizar esta variable serán analizados todos los ítems y sus diferentes apartados. De hecho se dan diferentes demandas cognitivas en muchos de los ítems.

De este modo queda definida otra Variable "Demanda cognitiva" y sus categorías “Conocer y reproducir", “Aplicar y relacionar" y "Razonar y reflexionar".

En cuanto al tipo de respuestas demandadas en los ítems también nos encontramos que en el Marco General de la evaluación final de Educación Primaria (MECD, 2015a) se establece una clasificación ya preestablecida para las pruebas:

- "Preguntas de respuesta cerrada, bajo el formato de elección múltiple, en las que solo una opción es correcta y las restantes se consideran erróneas.

- Preguntas de respuesta semiconstruida que incluyen varias preguntas de respuesta cerrada dicotómicas o solicitan al alumnado que complete frases o que relacione por medio de flechas diferentes términos o elementos.

- Preguntas de respuesta construida que exigen el desarrollo de procedimientos y la obtención de resultados. Este tipo de cuestiones 
contempla la necesidad de alcanzar un resultado único, aunque podría expresarse de distintas formas y describirse diferentes caminos para llegar al mismo. Tanto el procedimiento como el resultado han de ser valorados, para lo que hay que establecer diferentes niveles de ejecución en la respuesta en función del grado de desarrollo competencial evidenciado.

- Preguntas de respuesta abierta que admiten respuestas diversas, las cuales aun siendo correctas pueden diferir de unos alumnos o alumnas a otros $\mathrm{u}$ otras.” (MECD (2013), pág. 10)

La OCDE establece tres tipos de formatos según la respuesta demandada en los ítems. En este caso decido quedarme con la descripción dada por Marco General de la evaluación final de Educación Primaria (MECD, 2015a) ya que se ajusta a la estructura de los ítems. La caracterización hecha desde la OCDE no se ajusta al formato de los ítems y por lo tanto no nos aportaría información relevante.

Queda definida la Variable "Tipo de respuesta" y sus categorías "Cerrada", "Semiconstruida", "Construida" y "Abierta".

En estas pruebas de evaluación Marco General de la evaluación final de Educación Primaria (MECD, 2015a) establece una relación entre las pruebas y los estándares de aprendizaje descritos en el R.D. 126/2014, de 28 de febrero, por el que se establece el currículo básico de la Educación Primaria, ya que estos son los "referentes en la planificación de la concreción curricular y en la programación docente" Ministerio de Educación Ciencia y Deporte (2014). A su vez, establece que se agruparán en áreas y por bloques de contenido dentro de estas.

Debido a que los bloques de contenido van a ser una variable estudiada en este trabajo, en este caso nos basamos en los referentes dados desde la OCDE. En ellos se definen tres subcompetencias de la competencia científica que implican una serie de capacidades que permiten el desarrollo de dicha competencia, definiéndolas de esta manera:

- La primera subcompetencia sería "Explicar fenómenos científicamente" y es definida por la OCDE (2015) como la competencia necesaria para recordar y utilizar teorías, ideas, información y hechos; ofrecer explicaciones científicas, lo que requiere una comprensión de cómo este conocimiento se ha creado y el nivel 
de confianza que se puede tener en las afirmaciones científicas; conocer las formas y procedimientos estándares que se utilizan en la investigación científica para obtener dichos conocimientos y comprender su papel en la justificación de los conocimientos científicos.

- La segunda sería "Evaluar y diseñar investigaciones científicas" y es definida por la OCDE (2015) como la competencia que nos permite conocer los procedimientos básicos usados en el ámbito científico y la función de estos procedimientos a la hora de evaluar los nuevos avances científicos, permitiendo definir como una problemática de carácter científico puede ser resuelta.

- La tercera sería "Interpretar datos científicamente" y es definida por la OCDE (2015) como la competencia que nos permite dar argumentaciones basadas en datos científicos e interpretar si los datos dados en los argumentos de otras personas y por lo tanto sus argumentos son incorrectos.

Por otro lado, el Marco General de la evaluación final de Educación Primaria (MECD, 2015a) ya los pone como referentes a la hora de del desarrollo de la competencias básicas en ciencia y tecnología definidas en el "Interpretar datos científicamente". Por lo cual, las competencias descritas arriba tendrían total validez.

De esta manera dejamos definida la Variable "Capacidades competenciales" y sus categorías "Explicar fenómenos científicamente", "Evaluar y diseñar investigaciones científicas" y "Interpretar datos científicamente".

Por otro lado, también se evaluará el propio contenido científico. Para ello se utilizarán los bloques de contenido descritos por el Ministerio de Educación Ciencia y Deporte en el R.D. 126/2014, de 28 de febrero, por el que se establece el currículo básico de la Educación Primaria a excepción del bloque "Iniciación a la actividad científica" porque así lo establece Marco General de la evaluación final de Educación Primaria (MECD, 2015a). Quedando de la siguiente manera: "los seres vivos", "el ser humano y la salud", "la materia y la energía" y "la tecnología, los objetos y las máquinas".

El bloque de contenidos de los seres vivos "los seres vivos" puede ser definido como este conjunto de contenidos:

"Seres vivos, seres inertes. Diferenciación. Organización interna de los seres vivos. Estructura de los seres vivos: células, tejidos: tipos; órganos; aparatos 
y sistemas: principales características y funciones. Los seres vivos: Características, clasificación y tipos. Los animales vertebrados e invertebrados, características y clasificación. Las plantas: La estructura y fisiología de las plantas. La fotosíntesis y su importancia para la vida en la Tierra. Las relaciones entre los seres vivos. Cadenas alimentarías. Poblaciones, Comunidades y ecosistemas. Características y componentes de un ecosistema. Ecosistemas, pradera, charca, bosque, litoral y ciudad y los seres vivos. La biosfera, diferentes hábitats de los seres vivos. Respeto de las normas de uso, de seguridad y de mantenimiento de los instrumentos de observación y de los materiales de trabajo. Interés por la observación y el estudio riguroso de todos los seres vivos. Hábitos de respeto y cuidado hacia los seres vivos. Normas de prevención de riesgos. Uso de medios tecnológicos para el estudio de los seres vivos.” (MECD, 2014, pág. 19368)

El bloque de contenidos de los seres vivos "el ser humano y la salud" puede ser definido como este conjunto de contenidos:

"El cuerpo humano y su funcionamiento. Anatomía y fisiología. Aparatos y sistemas. Las funciones vitales en el ser humano: Función de relación (órganos de los sentidos, sistema nervioso, aparato locomotor). Función de nutrición (aparatos respiratorio, digestivo, circulatorio y excretor). Función de reproducción (aparato reproductor). Salud y enfermedad. Principales enfermedades que afectan a los aparatos y sistemas del organismo humano. Hábitos saludables para prevenir enfermedades La conducta responsable. Efectos nocivos del consumo de alcohol y drogas. Avances de la ciencia que mejoran la vida. Conocimiento de actuaciones básicas de primeros auxilios. Conocimiento de sí mismo y los demás. La identidad y la autonomía personal. La relación con los demás. La toma de decisiones: criterios y consecuencias. La resolución pacífica de conflictos. La igualdad entre hombres y mujeres.” (MECD, 2014, pág. 19369)

El bloque de contenidos de los seres vivos "la materia y la energía" puede ser definido como este conjunto de contenidos:

"Estudio y clasificación de algunos materiales por sus propiedades. Utilidad de algunos avances, productos y materiales para el progreso de la sociedad. 
Diferentes procedimientos para la medida de la masa y el volumen de un cuerpo. Explicación de fenómenos físicos observables en términos de diferencias de densidad. La flotabilidad en un medio líquido. Predicción de cambios en el movimiento o en la forma de los cuerpos por efecto de las fuerzas. Concepto de energía. Diferentes formas de energía. Fuentes de energía y materias primas: su origen. Energías renovables y no renovables. La luz como fuente de energía. Electricidad: la corriente eléctrica. Circuitos eléctricos. Magnetismo: el magnetismo terrestre. El imán: la brújula. Planificación y realización de experiencias diversas para estudiar las propiedades de materiales de uso común y su comportamiento ante la luz, el sonido, el calor, la humedad y la electricidad. Observación de algunos fenómenos de naturaleza eléctrica y sus efectos (luz y calor). Atracción y repulsión de cargas eléctricas. Separación de componentes de una mezcla mediante destilación, filtración, evaporación o disolución. Reacciones químicas: la combustión, la oxidación y la fermentación. Utilidad de algunos avances, productos y materiales para la sociedad. Fuentes de energías renovables y no renovables. El desarrollo energético, sostenible y equitativo.” (MECD, 2014, pág. 19370)

El bloque de contenidos de los seres vivos "la tecnología, los objetos y las máquinas" puede ser definido como este conjunto de contenidos:

"Máquinas y aparatos. Tipos de máquinas en la vida cotidiana y su utilidad. Análisis de operadores y utilización en la construcción de un aparato. Construcción de estructuras sencillas que cumplan una función o condición para resolver un problema a partir de piezas moduladas. La electricidad en el desarrollo de las máquinas. Elementos de los circuitos eléctricos. Efectos de la electricidad. Conductores y aislantes. La relación entre electricidad y magnetismo. La ciencia: presente y futuro de la sociedad. Beneficios y riesgos de las tecnologías y productos. Importantes descubrimientos e inventos. Tratamiento de textos. Búsqueda guiada de información en la red. Control del tiempo y uso responsable de las tecnologías de la información y la comunicación.” (MECD, 2014, pág. 19371) 
A la hora de analizar esta variable solo se tendrá en cuenta la que tenga mayor importancia en el ítem. Si no se hiciera así se darían varios de estos bloques de contenido en un mismo ítem, aunque en realidad es uno de ellos el que tiene mayor peso dentro del mismo. Este es el mismo procedimiento que utiliza el programa PISA para la concreción de sus ítems de evaluación.

De esta manera dejamos definida la Variable "Contenido" y sus categorías "los seres vivos", "el ser humano y la salud", "la materia y la energía" y "la tecnología, los objetos y las máquinas". 


\section{Instrumento y Resultados}

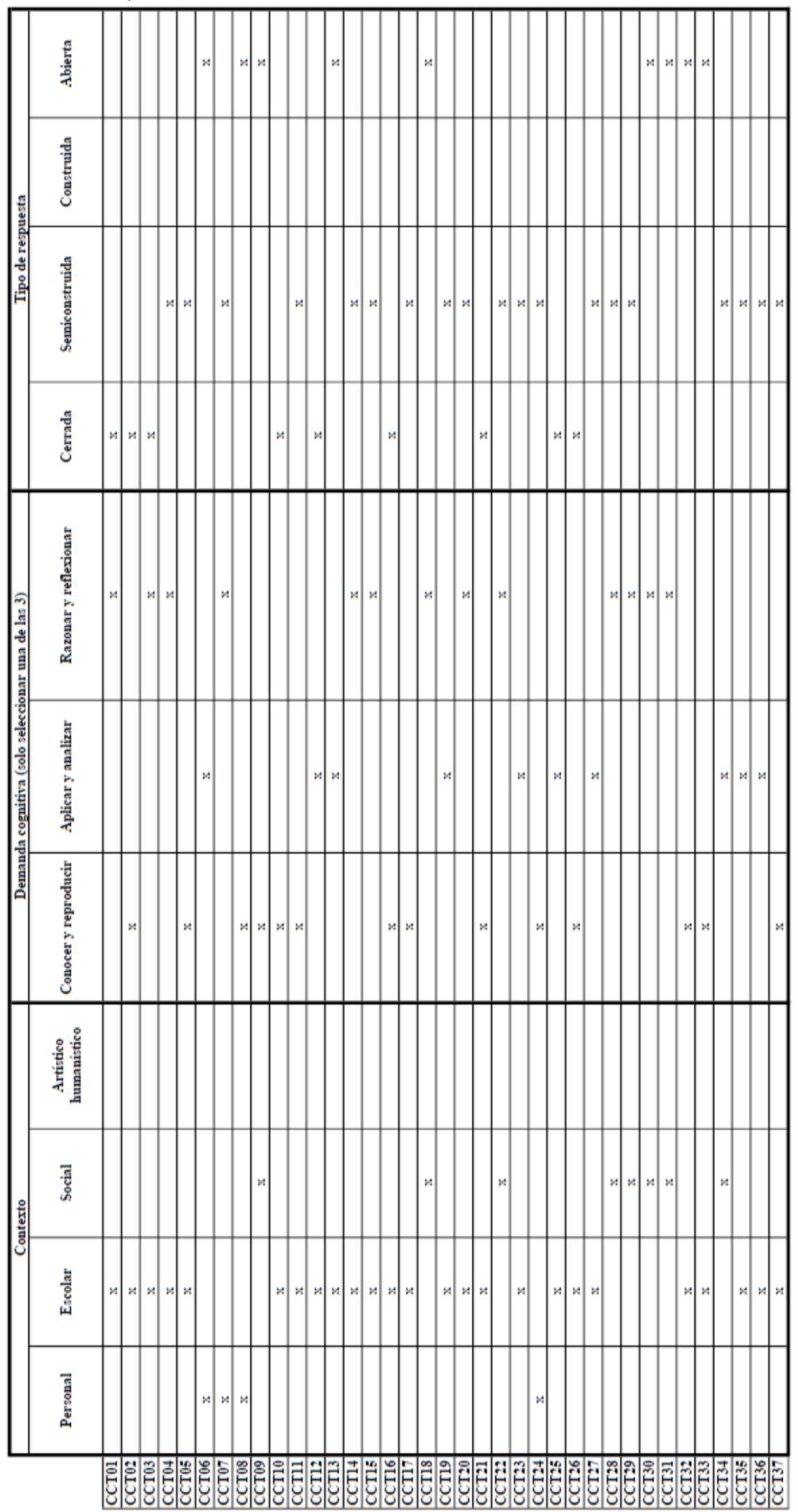

Figura 1 Parrilla de observación contestada hoja 1. En esta figura se puede ver la primera hoja de la parrilla de observación. Las casillas señaladas con una "x" corresponden a las observaciones realizadas por los observadores tras el consenso. 


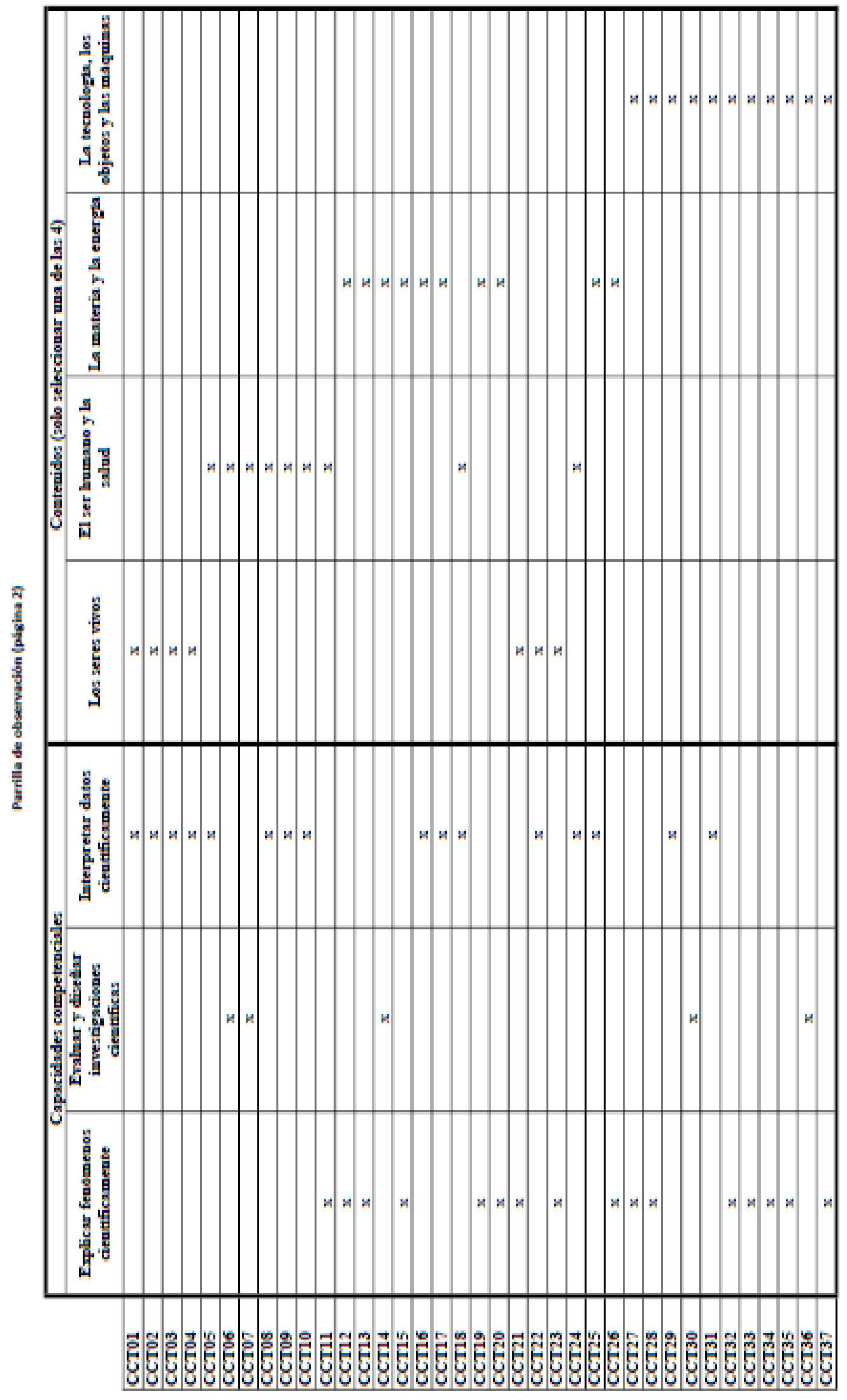

Figura 2. Parrilla de observación contestada hoja 2. En esta figura se puede ver la segunda hoja de la parrilla de observación. Las casillas señaladas con una " $\mathrm{x}$ " corresponden a las observaciones realizadas por los observadores tras el consenso. 


\section{Sistema de variables}

\begin{tabular}{|c|c|c|}
\hline Variables & Valores & Palabras clave \\
\hline \multirow{4}{*}{ Contexto } & Personal & Yo mismo, familia y amigos. \\
\hline & Escolar & Vida escolar y compañeros de clase. \\
\hline & Social & Barrio, localidad y sociedad. \\
\hline & Artistico humanistico & Mundo artístico y Ciencias Sociales. \\
\hline \multirow{3}{*}{ Demanda cognitiva } & Conocer y reproducir & $\begin{array}{l}\text { Nombrar, definir, encontrar, mostrar, listar, enumerar, contar, recordar, reconocer, } \\
\text { localizar, reproducir, relatar, relacionar, caracterizar, explicar, ilustrar, extractar, resumir, } \\
\text { completar, traducir a otros términos, relacionar, describir, enumerar, listar, poner } \\
\text { ejemplos. }\end{array}$ \\
\hline & Aplicar y analizar & $\begin{array}{l}\text { Clasificar, contrastar, comparar, relacionar, enumerar, listar, vincular, distinguir, } \\
\text { diferenciar, ordenar, asociar, establecer, utilizar, representar, ilustrar, interpretar, modelar, } \\
\text { relacionar, explicar, relatar, utilizar conocimiento, demostrar, solucionar, identificar, } \\
\text { expresar. }\end{array}$ \\
\hline & Razonar y reflexionar & $\begin{array}{l}\text { Concluir, determinar, reformular, analizar, diseñar, imaginar, inventar, planificar, predecir, } \\
\text { proponer, adaptar, estimar, desarrollar, extrapolar, criticar, juzgar, recomendar, } \\
\text { establecer criterios y/o limites, opinar, valorar, cuestionar. }\end{array}$ \\
\hline \multirow{4}{*}{ Tipo de respuesta } & Cerrada & Elección múltiple y una correcta. \\
\hline & Semiconstruida & Dicotómicas, complete frases y relacione con flechas. \\
\hline & Construida & Desarrollo de procedimientos y obtención de resultados. \\
\hline & Abierta & Respuestas diversas y correctas. \\
\hline \multirow{3}{*}{ Capacidades competenciales } & $\begin{array}{c}\text { Explicar fenómenos } \\
\text { cientificamente }\end{array}$ & Reconocer, ofrecer y evaluar explicaciones de fenómenos naturales y tecnológicos. \\
\hline & \begin{tabular}{|c|} 
Evaluar y diseñar \\
investigaciones cientificas
\end{tabular} & Describir y valorar investigaciones cientificas y proponer heuristicos cientificos. \\
\hline & $\begin{array}{l}\text { Interpretar datos } \\
\text { cientificamente }\end{array}$ & Analizar datos, afirmaciones y argumentos y extraer conclusiones cientificas apropiadas. \\
\hline
\end{tabular}

Figura 3. Variables, valores y palabras clave hoja 1. En esta figura se puede ver el sistema de variables entregado, a los observadores, correspondiente a las variables Contexto, Demanda cognitiva, Tipo de respuestas y Capacidades competenciales.

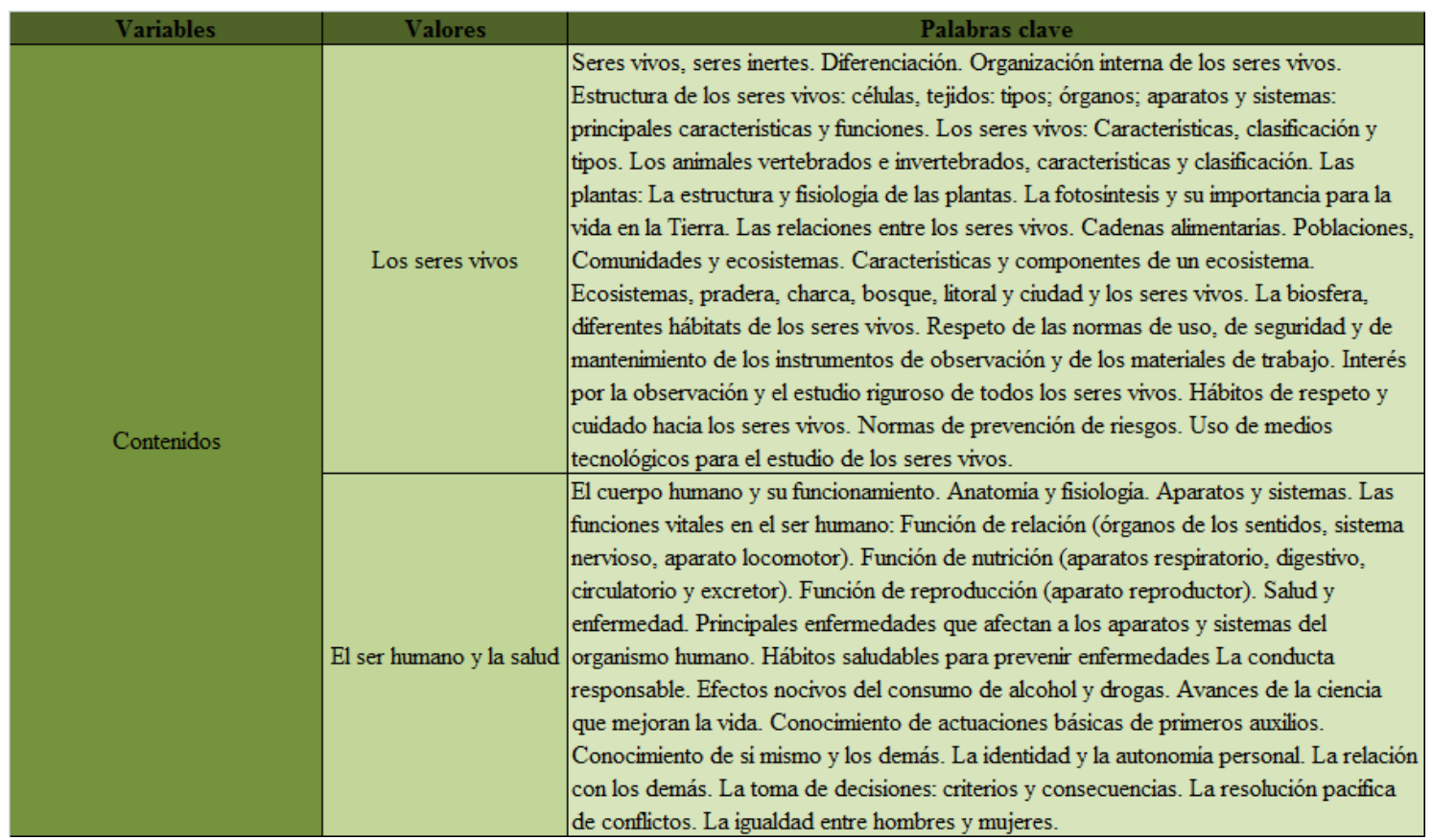

Figura 4. Variables, valores y palabras clave hoja 2. En esta figura se puede ver el sistema de variables entregado, a los observadores, correspondiente a la variable Contenido y sus valores Los seres vivos y El ser humano y la salud. 


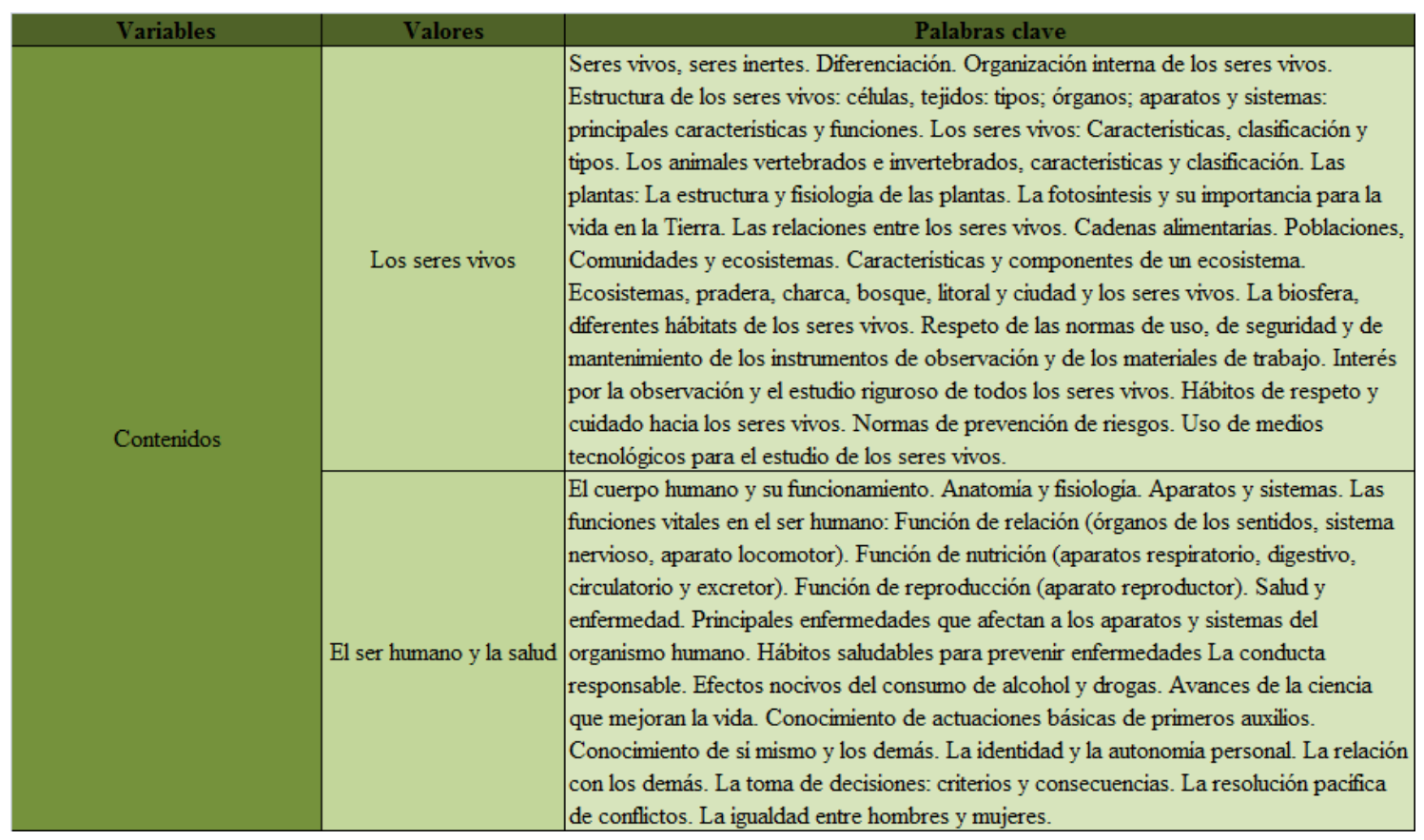

Figura 5. Variables, categorías y palabras claves hoja 3. En esta figura se pueden ver la tabla de Variables, categorías y palabras claves entregada, a los observadores, correspondiente a la variable Contenido y sus valores La materia y la energía y La tecnología, los objetos y las máquinas. 
4. Ejemplo del análisis en un ítem (tarea) específica

\section{UN PASEO POR EL BOSOUE}

Alejandra ha salido de excursión con su clase. Han pasado la mañana paseando por un bosque que hay cerca de su ciudad. Se ha llevado su cámara y ha sacado estas fotografías:

A)

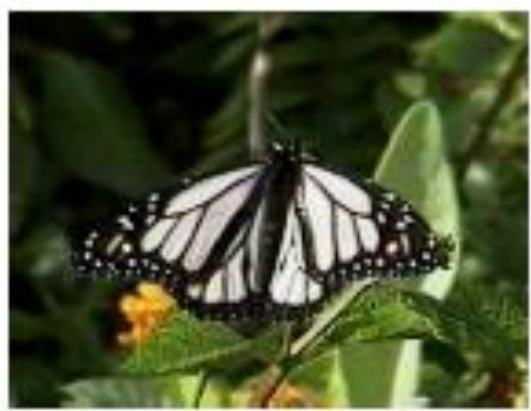

c)

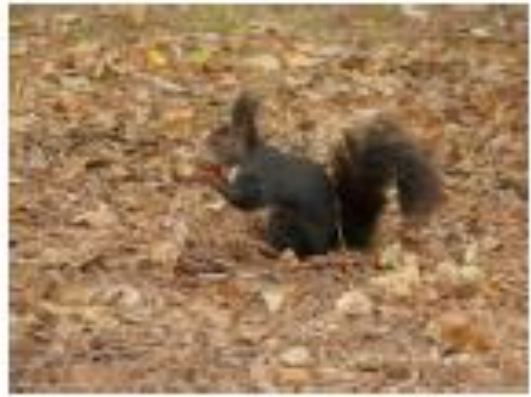

E

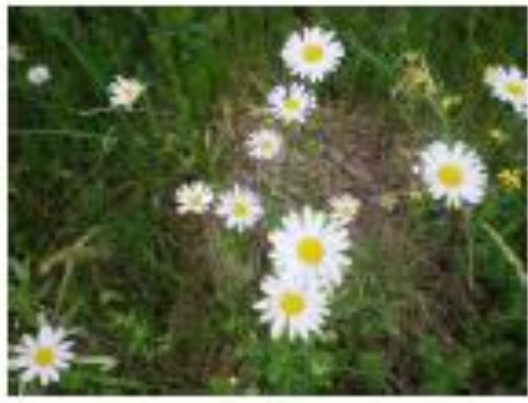

B)

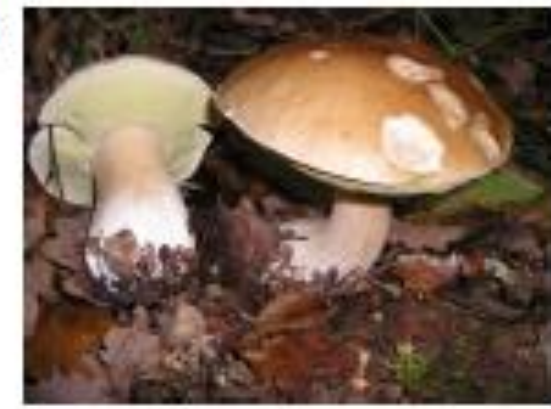

D)

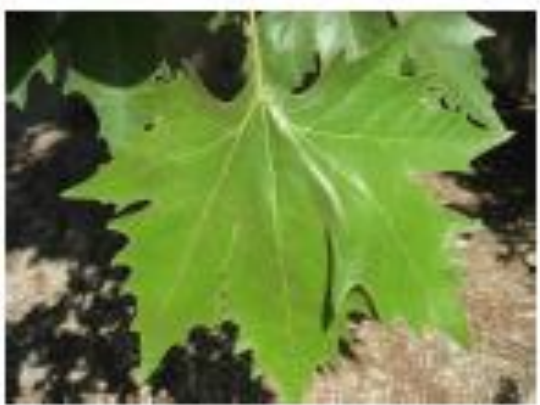

Figura 6. Situación problemática un paseo por el bosque. Se present una situación problemática que sirve de punto de partida para los ítems. 
1. Ahora Alejandra quiere clasificar las fotos que ha sacado, y a cada una le va a poner una etiqueta que ponga una de estas tres cosas: "Reino Animal", "Reino Vegetal" o "Reino de los Hongos".

Rellena la siguiente tabla para ayudar a Alejandra.

\begin{tabular}{|c|c|}
\hline Fotografía & Reino \\
\hline A & \\
\hline B & \\
\hline C & \\
\hline D & \\
\hline E & \\
\hline
\end{tabular}

Figura 7. Ítem CCT01. Este ítem demanda al alumnado una clasificación de los seres vivos.

A continuación, ejemplificamos uno de los ítems.

En cuanto a la variable Contexto, los jueces, han considerado que se trata de un contexto Escolar, ya que se trata de un ejercicio planteado a una alumna dentro de una actividad extraescolar (excursión).

Han catalogado este ítem con la demanda cognitiva Razonar y reflexionar debido a que incluye el uso de las evidencias como las fotografías y de la comprensión científica para analizar, sintetizar y generalizar, a menudo en situaciones no familiares y contextos complejos como una salida al bosque.

El tipo de respuesta ha sido considerada como cerrada. Esto se debe a que solo hay una respuesta correcta y las demás deben de ser descartadas.

La capacidad competencial observada es Interpretar datos científicamente. Esta competencia nos permite dar argumentaciones basadas en datos científicos como las fotografías, en este caso los argumentos no se piden expresados explícitamente pero el alumnado debe de elaborarlas de forma interna para responder.

Por último, el contenido utilizado corresponde a los seres vivos ya que consideran que aparecen diversos seres vivos que deben ser clasificados y diferenciados. 